\title{
A Review: Prognostics and Health Management in Automotive and Aerospace
}

\author{
Van Duc Nguyen ${ }^{\mathrm{a}}$, Marios Kefalas ${ }^{\mathrm{a}}$, Kaifeng Yang ${ }^{\mathrm{a}}$, Asteris Apostolidis ${ }^{\mathrm{b}}$, Markus Olhofer ${ }^{\mathrm{c}}$, Steffen Limmer ${ }^{\mathrm{c}}$, and Thomas \\ Bäck $^{\mathrm{a}}$ \\ ${ }^{a}$ LIACS, Leiden University, Leiden, 2333 CA, The Netherlands \\ d.v.nguyen@liacs.leidenuniv.nl \\ m.kefalas@liacs.leidenuniv.nl \\ k.yang@liacs.leidenuniv.nl \\ t.h.w.baeck@liacs.leidenuniv.nl \\ b KLM Royal Dutch Airlines, 1181 GP Amstelveen The Netherlands \\ Asteris.Apostolidis@klm.com \\ c Honda Research Institute Europe GmbH, 63073 Offenbach am Main, Germany \\ Steffen.Limmer@honda-ri.de \\ markus.olhofer@honda-ri.de
}

\begin{abstract}
Prognostics and Health Management (PHM) attracts increasing interest of many researchers due to its potentially important applications in diverse disciplines and industries. In general, PHM systems use real-time and historical state information of subsystems and components of the operating systems to provide actionable information, enabling intelligent decision-making for improved performance, safety, reliability, and maintainability. Every year, a substantial number of papers in this area including theory and practical applications, appear in academic journals, conference proceedings and technical reports. This paper aims to summarize and review researches, developments and recent contributions in PHM for automotive- and aerospace industries. It can also be considered as the starting point for researchers and practitioners in general to assist them through PHM implementation and help them to accomplish their work more easily.
\end{abstract}

\section{INTRODUCTION}

\subsection{General introduction}

At 11:03 Eastern Daylight Time (EDT), Southwest Airlines Flight 1380 from New York to Dallas, was at about flight level (FL) 320 (an altitude of approximately 32,000 feet) and climbing when the left engine failed. As a result most of the

\footnotetext{
Duc Van Nguyen \& Marios Kefalas et al. This is an open-access article distributed under the terms of the Creative Commons Attribution 3.0 United States License, which permits unrestricted use, distribution, and reproduction in any medium, provided the original author and source are credited.
}

engine inlet and parts of the cowling broke off. Fragments from the inlet and cowling struck the leading edge of the wing and fuselage, causing a rapid depressurization. After investigations, the reason was found to be failure of a single fan blade, due to a fatigue crack (Accident: Southwest B737 near Philadelphia on Apr 17th 2018, uncontained engine failure takes out passenger window, n.d.). On 30th of September 2017 Air France Flight 66 from Paris to Los Angeles suffered an uncontained engine failure and made an emergency landing at Goose Bay Airport, Canada. Investigations indicated that the engine's fan hub had detached and dragged the air inlet with it during the flight (Incident: France A388 over Greenland on Sep 30th 2017, uncontained engine failure, fan and engine inlet separated, n.d.). We all know that, unfortunately, failure has never been completely prevented although much money has been spent for equipment maintenance.

According to the annual reports of the Royal Dutch Airlines $(\mathrm{KLM})^{1}$, the maintenance costs from 2013 to 2017 are 669, 665, 934, 1009, and 994 million euro, respectively. These correspond to about $11 \%$ to $18 \%$ of the operation costs. In September 2003, the Commission of the European Community reported that repair and maintenance accounts for $40 \%$ of the total lifetime costs of vehicle ownership (Taie, Diab, $\&$ ElHelw, 2012). The consequent costs due to the equipment failure are high. DHL has estimated that an AOG (Aircraft On Ground) due to technical reasons for an A380 Airbus, costs as much as 925.000 euro per day ${ }^{2}$. In the worst cases, the con-

\footnotetext{
${ }^{1}$ https://www.klm.com/corporate/en/publications/2015_Annual_Report.html ${ }^{2}$ Source: Airbus China
} 
sequent costs could not be fully evaluated if the equipment failure led to a bad accident.

Prognostics and Health Management (PHM), therefore, has emerged over recent years as an approach and methodology that has a great impact in all industries. PHM is an engineering discipline that aims at minimizing maintenance cost by the assessment, prognosis, diagnosis, and health management of engineered systems. With an increasing prevalence of smart sensing and with more powerful computing, PHM has been gaining popularity across a growing spectrum of industry such as aerospace, smart manufacturing, transportation, and power generation (Ekwaro-Osire, Stephen, Alemayehu, Fisseha M, \& Carlos Gonalves, Aparecido, 2017). Regardless of application, one common expectation of PHM is its capability to translate raw data into actionable information to facilitate maintenance decision making. Sometimes, PHM is referred to as system health management (SHM), integrated systems health management (ISHM), vehicle health management system (VHMS) or engine health management (EHM).

In general, PHM provides for viewing overall health state of machines or complex systems and assists in making correct decisions on machine or system maintenance. A robust PHM system should be able to detect incipient component or system fault, perform failure diagnostics, failure prognostics, and health management. Failure prognostics is the heart of PHM. It refers specifically to the phase involved with predicting future behavior and the system's useful lifetime left in terms of current operating state and the scheduling of required maintenance actions to maintain system health (Vachtsevanos, Lewis, Roemer, Hess, \& Wu, 2006). The useful lifetime left is often called the 'Remaining Useful Life (RUL)'. RUL is typically a random variable and unknown, and as such it must be estimated from available sources of information such as the information obtained in condition and health monitoring(Si, Wang, Hu, \& Zhou, 2011). The main implementation steps for PHM consist of; i) defining critical component(s), ii) appropriate sensor selection for condition monitoring, iii) prognostics feature evaluation under data analysis and iv) prognostics methodology and tool evaluation matrices(Atamuradov, Medjaher, Dersin, Lamoureux, \& Zerhouni, 2017).

PHM applications can be classified into two main categories based on how the PHM is applied to the system or to the product (Sutharssan, Stoyanov, Bailey, \& Yin, 2015): i) real-time PHM (sometimes referred as online PHM or on-board health monitoring), ii) off-line PHM. Most of the safety critical and mission critical applications require the real-time PHM. Usually modern aircrafts, automobiles and so on have substantial on-board monitoring capability that is based on the use of data from real-time sensors. For example, an electric car provides the range which can be achieved with the current battery state of charge based on the real-time monitoring of the battery. Another example is the autonomous unmanned vehicles, which have embedded real- time on-board PHM used to re-plan the mission and reconfigure the controls based on the health diagnostic and prognostic information. Such capability requires the evaluation of the current state of the health and also a prediction of the future state of the component/ systems health (Tang et al., 2008; Sutharssan et al., 2015).

Approaches dealing with PHM are generally classified into four categories: reliability based, model-based, data-driven and hybrid. Each approach has its own advantages and drawbacks. This topic will be discussed in more detail later on in this review.

\subsection{Existing Review Articles on PHM}

There are a few review papers on PHM approaches and applications. Hereby, we list some examples by following the order of appearance, from the oldest to the newest.

Jardine et al.(Jardine, Lin, \& Banjevic, 2006) summarized and reviewed research and developments in diagnostics and prognostics of mechanical systems implementing Condition Based Maintenance (CBM) with emphasis on models, algorithms and technologies for data processing and maintenance decision-making. Realizing the increasing trend of using multiple sensors in condition monitoring, the authors discussed different techniques for multiple sensor data fusion.

Wheeler et al.(Wheeler, Kurtoglu, \& Poll, 2009) provided a review over PHM user objectives and how they are related to metrics commonly used in diagnostics and prognostics. In this paper, authors identified critical gaps within the user objectives and the engineering development. A detailed survey on the objectives of different users of health management systems was presented. These user objectives were then mapped to the metrics typically encountered in the development and testing of two main systems health management functions: diagnosis and prognosis. They found that although the metrics associated with diagnostic and prognostic algorithm and system performance positively impact the user community, there were gaps within the diagnostic and prognostic metrics.

Si et al.(Si et al., 2011) reviewed the statistical data-driven approaches for RUL estimation. Here authors reported the up-to-date modeling developments for estimating the RUL. The review was centered on statistical data driven approaches which rely only on available past observed data and statistical models. The approaches are classified into two broad types of models, that is, models that rely on directly observed state information of the asset, and those do not. They systematically reviewed the models and approaches reported in the literature and finally highlighted future research challenges namely i) development of a RUL estimation model based on very few or no data situations. ii) data fusion where multi-dimensional condition monitoring (CM) input data must be dealt with. Be- 
cause it imposes a severe challenge to threshold-based models which are mostly established under a single threshold level. iii) development of a model the influence of external environmental variables. This is a complicated issue since those variables will impact on the observed $\mathrm{CM}$ variables which in turn will influence the RUL estimation. If it is not done properly, overfitting can occur, which may reduce the robustness of the developed estimation model. iv) development of a model which can deal with multiple failure modes for a single component.

Lee et al.(Lee et al., 2014) reviewed research on PHM design for rotary machinery systems. This paper provides a review of the PHM field, followed by an introduction of a systematic PHM design methodology for converting data to prognostics information. This methodology includes procedures for identifying critical components, as well as tools for selecting the most appropriate algorithms for specific applications. Visualization tools are presented for displaying prognostics information in an appropriate fashion for quick and accurate decision making. Industrial case studies are included in this paper to show how this methodology can help in the design of an effective PHM system.

A specific software, which we will be referring to in the rest of this study, is the Commercial Modular Aero-Propulsion System Simulation (C-MAPSS) and its datasets. This is a run-to-failure software and the datasets are generated from a turbofan engine simulation model (Saxena \& Goebel, 2008). The dataset was first published by NASAs Prognostics Center of Excellence (PCoE) in 2008. The original purpose of generating this dataset was to use it in a data challenge competition in PHM08 conference, where PHM researchers were invited to develop prognostic methods as part of the competition. However, since then, these datasets have been widely used by researchers around the world for developing prognostic approaches and results in many publications. Nonetheless, during the first six years, it was difficult for the users to suitably compare their results due to the absence of performance benchmarking results and common misunderstandings in interpreting the relationships between these datasets. In 2014, Ramasso et al. (Ramasso \& Saxena, 2014) wrote a review paper to summarize these approaches and analyzed them to understand why some approaches worked better than others, how did researchers use these datasets to compare their methods, and what were the difficulties faced, so necessary improvements can be made to these datasets to make them more useful. The paper establishes public knowledge that helps users in prognostic algorithm development and aids in fulfilling the underlying intent of data repository to facilitate algorithm benchmarking development.

Liu et al.(W. Liu et al., 2015) reviewed the structure healthy condition monitoring and fault diagnosis methods in wind turbines. In this paper, authors reviewed the structure of wind turbines and analyzed the different components of wind turbines in order to detect the faults that may happen. They mainly reviewed fault diagnosis methods of wind turbines in the last three years (up to 2015). Some research results on diagnosing wind turbine components were analyzed, such as time-frequency analysis methods, vibration based methods, voltage and current based methods, etc. The advantages and drawbacks of these methods were compared in detail in order to find the most suitable methods. The main purpose of this paper was to supply some information on structure healthy condition monitoring and fault diagnosis in wind turbines for related researchers.

Two independent groups reviewed the data-driven approach and algorithms for PHM (Tsui, Chen, Zhou, Hai, \& Wang, 2015; Sutharssan et al., 2015). Tsui et al. provided main concepts and mathematical formulations that help readers to quickly catch the key ideas and guidelines of each method. They also showed three examples to illustrate the implementation of PHM. The first example was to identify fault diagnosis on gear crack development. The best classification accuracy used weighted $k$ nearest neighbor method and was near $100 \%$, which was very beneficial for early warning of potential gearbox malfunction. The second example was to predict RUL of rotational bearings. The results showed that the prediction based on the data-driven method was acceptably accurate, which provides very informative warnings on the potential failures. The last example was to predict RUL of Lithium-Ion batteries using Particle Filter. In the experiment, batteries were tested with full charging and discharging cycles, under the constant-current/constant voltage mode. The results showed that the prediction was better and the probability density function (PDF) of RUL was narrower at the later stage of the batterys life.

In parallel, Sutharssan et al.(Sutharssan et al., 2015) aimed at reviewing the structure, state-of-the-art, and classification of algorithms and methods used to underpin different existing data-driven PHM approaches. This paper discussed different algorithms and mathematical models under different data-driven PHM approaches. They showed that each approach and algorithm has its own advantages and disadvantages depending on the application, availability of the historical data, system specific knowledge, programmability and so on. PHM applications also have many different individual processes such as noise reduction, anomaly detection, fault isolation and monitoring, state estimation, lifetime prediction and so on. They concluded that the selection of the approach and algorithm for each process of a PHM application plays a key role and is an important factor for the accuracy of the overall PHM methodology.

Coble et al.(Coble, Ramuhalli, Bond, Hines, \& Ipadhyaya, 2015) reviewed PHM applications in nuclear power plants. Here authors highlighted the key research needs and techni- 
cal gaps that must be addressed in order to fully realize the benefits of PHM in nuclear facilities. They also reviewed the state of the art and the state of practice of prognostics and health management for nuclear power systems. They concluded that research, development, and deployment of PHM for nuclear power systems have largely lagged behind other safety-critical industries.

Jung et al.(Jung \& Ismail, 2015) attempted to provide an overview about PHM trend and direction of PHM in automotive industry. However, authors failed to do so. They just simply listed some publications of PHM on the battery, engine, antilock braking system and electric power steering system. But they did not review and discuss anything about methods and results.

Vogl et al.(Vogl, Weiss, \& Helu, 2016) had a review of diagnostic and prognostic capabilities and best practices for manufacturing. This paper reviews the challenges, needs, methods, and best practices for PHM within manufacturing systems. This includes PHM system development of numerous areas highlighted by diagnostics, prognostics, dependability analysis, data management, and business. They pointed out that the challenges and needs that must be overcome for the widespread realization of PHM within manufacturing. Based on current capabilities, the critical challenges are real-time diagnostic and prognostic methods, standards for PHM system evaluation, and the integration of data within user-friendly PHM systems. Specifically, this PHM system be both reliable and flexible for use with multiple data sources.

Elattar et al.(Elattar, Elminir, \& Riad, 2016) wrote a literature review in prognostics in general. To the best of our knowledge, this is the first comprehensive vision about prognostics as a part of PHM in a single literature review paper. Authors focused on reviewing prognostics benefits, approaches, applications, and challenges. They gathered a lot of sparse information about prognostics and combined all of these information together to present an integrated work that shows the importance of prognostics and its influencing rule in PHM. They also clarified how the maintenance strategies can shift from fail and fix to predict and prevent based on the proactivity in prognostics and how prognostics is the main building block in CBM. They discussed the prognostics approaches, their advantages and disadvantages, and how to use the suitable technique according to the prognostics problem definition. They also presented a lot of prognostics applications which have been already deployed or are just an experiment. Finally, they addressed the more challenging aspects in prognostics and how the research community is trying to resolve these challenges. This paper can be considered as a starting point for new prognostics researchers.

Atamuradov et al.(Atamuradov et al., 2017) had a review of implementation and tools evaluation of PHM for maintenance practitioners. Authors presented a general view of PHM and its steps to provide prior knowledge for users, reviewed different PHM approaches under model-based, data-driven and hybrid models, and discussed their merits and drawbacks. They also reviewed previous and on-going research in bogie components PHM to highlight problems faced in the railway industry. As a result of PHM literature review on bogie components, they noticed that nearly all research conducted in bogie health assessment is mostly limited to diagnostics rather than prognostics tasks. Since railway vehicle bogies are critical components, research on prognostics for asset health management is also crucial to provide a safe and comfortable ride for customers.

The successful PHM applications in the industry require the contributions from not only the field of reliability engineering and maintenance scheduling, but also the field of manufacturing engineering. In recent 20 years, production systems of advanced manufacturing paradigms (e.g. mass customization, reconfigurable manufacturing, sustainable manufacturing and service-oriented manufacturing) have been developed to exceed the traditional mass production paradigm. The reasons that make system health management especially difficult include, individual machine deterioration, different system structures, diverse production characteristics and exponential scheduling complexity. To address these gaps, Xia et al. (Xia et al., 2018) provided a review of the PHM work focusing on prognostics approaches for asset health, and maintenance policies for more "informed" decisions. This paper addresses recent advances in PHM for advanced manufacturing paradigms, to forecast health trends, avoid production breakdowns, reduce maintenance cost and achieve rapid decision making. Furthermore, an in-depth look at future research interests in this field is also provided.

Here we would like to introduce a comprehensive vision about PHM with emphasizing the previous and on-going researches in PHM for automotive- and aerospace industries. This paper can also be considered as the starting point for researchers and practitioners to assist them through PHM implementation and help them to accomplish their duty more easily.

The remainder of this review is organized as follows; section II discusses failure modes and failure mechanisms, section III diagnostics and prognostics, section IV introduces PHM methods and section V covers the performance metrics. Section VI and VII review PHM applications in the automotive and aerospace industry, respectively and in section VIII we conclude this review.

\section{FAILURE MODE AND FAILURE MECHANISMS}

An important part in PHM system is to clarify and identify failure mode and failure mechanism. Failure mode is the manner in which a system or component functionally fails. It describes to what extent a certain function cannot be fulfilled 
anymore. Therefore, failure does not always imply the real physical failure of a part, like fracture or melting, but could also be the result of extensive deformation leading to rubbing or seizure of a rotating part. Moreover, the definition of failure depends on what level is considered. Failure of a specific part or subsystem does not automatically imply that the complete system fails. For instance, a plant equipped with several pumps does not stop when only one pump fails. In that case, a failure occurs on the subsystem level (pump), but no failure occurs on the system level (plant). In short, the failure modes are generally related to the performance requirements of the system (Tinga, 2013).

On the other hand, failure mechanisms are physical, chemical, thermodynamic or other processes that result in failure. In PHM, knowing the failure mechanisms is a must to develop model-based methods and is crucial to identify and select features in the data-driven methods. Failure mechanisms are categorized as either overload or wear-out mechanisms. Overload failure arises because of a single load condition, which exceeds a fundamental strength property. Wearout failure arises as a result of cumulative damage related to loads applied over an extended time (Pecht \& Jie Gu, 2009). Knowledge on these mechanisms, and especially the effect of the governing loads on failure, are essential to understand why, how and when components fail and how this can be prevented. They are critically important in the PHM. The interested reader can find more details to failure mechanisms in (Tinga, 2013). This book provides an overview of the most important failure mechanisms. That includes static overload, deformation, corrosion, fatigue, creep, wear, melting, thermal degradation, electric failures, and radiative failures.

\section{Diagnostics AND PROgnOSTICS}

Diagnostics and prognostics are processes of assessment of a system's health. Diagnostics is an assessment about the current (and past) health of a system based on observed symptoms. It deals with fault detection, isolation and identification when a fault occurs. Fault isolation locates the fault to a specific component or area of a structure. Fault identification determines the root cause of the fault. Often, these analyses are completed in concert with each other; when an anomaly is detected, the diagnostic system typically determines both the location and cause of the fault given the available fault symptoms. Fault symptoms include the signatures that may help diagnose the fault, including sensed data, features extracted from sensed data, monitoring system residuals, and anomaly detection results (Coble et al., 2015). Diagnostic capabilities traditionally have been applied at or between the initial detection of a system, component, or sub-component failure and complete system catastrophic failure. In order to maximize the benefits of continued operational life of a system or subsystem component, maintenance often will be delayed until the early incipient fault progresses to a more severe state but before an actual failure event. Practitioners reasoned that if it were possible to use existing data and data sources to diagnose failed components, why would it not be possible to detect and monitor the onset of failure, thus preventing failures before they actually hamper the ability of the operating system to perform its functions. By doing this, mission reliability would be increased greatly, maintenance actions would be scheduled better to reduce system down time, and a dramatic decrease in life-cycle costs could be realized. More recent diagnostic technologies enable the detection in much earlier fault stages. The increase in this diagnostic capability naturally has evolved into something more: the desire for prognosis (Dong \& He, 2007).

Prognostics is an assessment of the future health, it is a task to determine whether a fault is impending and estimate how soon and how likely a fault will occur. If an operator has the will to continue to operate a system and/or component with a known, detected incipient fault present, he or she will want to ensure that this can be done safely and will want to know how much useful lifetime remains at any point along this particular failure progression timeline. This is the specific domain of real predictive prognosis, being able to accurately predict the RUL along a specific failure progression timeline for a particular system or component. However, do not confuse prognostic with RUL prediction. Because besides the RUL prediction, a comprehensive prognostic should be able to quickly and efficiently isolate the root cause of failures. In this sense, if fault/ failure predictions can be made, the allocation of replacement parts or refurbishment actions can be scheduled in an optimal fashion to reduce the overall operational and maintenance logistic footprints. From the fault isolation perspective, maximizing system availability and minimizing downtime through more efficient troubleshooting efforts is the primary objective (Vachtsevanos et al., 2006).

\section{PHM APPROACHES}

Commonly, prognostics approaches are classified into four types (Elattar et al., 2016) namely i) reliability-based approaches, ii) model-based approaches, iii) data-driven approaches, and iv) hybrid approaches. Each approach has its own merits and limitations. Nonetheless, generally speaking, the complexity, cost, and accuracy of prognostics techniques is usually inversely proportional to its applicability. Increasing prognostics algorithm accuracy with low cost and complexity is a big challenge. The prognostics system developers can benefit from this classification in the prognostics approaches selection based on available data and their knowledge about the engineer system. A key point about prognostics approaches classification is to build a way to obtain a standard methodology for prognostics applications development within a standard framework. 


\subsection{Reliability-based Approaches}

Experienced-based prognostics, life usage model, or statistical reliability-based approach are different names for the same approach. These approaches are the simplest form of fault prognostics as they require less detailed information than other prognostic approaches. They are based on the distribution of event records of a population of identical items. Many traditional reliability approaches such as exponential, Weibull, and log-normal distributions have been used to model asset reliability. In practical applications, reliabilitybased approaches can be implemented when historical repair and failure data are available. These approaches do not consider the failure indication (degradation) of an asset when predicting asset life (Gorjian, Ma, Mittinty, Yarlagadda, \& Sun, 2010). In addition, these approaches are used mainly for uncritical, unmonitored components that do not have a physical model and are mass produced. We, therefore, exclude reviewing applications of these approaches in this paper.

\subsection{Model-based Approaches}

The model-based method, sometimes referred as physicsbased method, is the most important approach in PHM because of its accuracy, precision, and real-time performance (Elattar et al., 2016). It is a deterministic method and allows the estimation and the prediction of the dynamic states. In this approach, a physical/mathematical model for the system or component is developed. This model is a real-time, mathematical representation of the system that is able to predict the system degradation and failures. Additionally, it is able to detect shifts from the nominal conditions when a simulation based on the model runs in parallel to the actual process. To establish this model, a thorough understanding of the physics of the system/component is required and such a model's reliability often decreases as the system complexity increases. However, model-based methods do not require a large amount of data and especially the data of the failure events. Besides some physics-based models that are developed based on physical principles/laws, the most common model-based methods are Kalman filters (KF), extended Kalman filters (EKF), unscrented Kalman filters (UKF), and particle filter (PF).

Kalman filters were introduced as a fault isolation and assessment technique for relative aircraft engine performance diagnostics in the late 1970s and early 1980s (Simon, 2008). More widely used by engineers and other physical scientists, filtering problems are mathematical models for state estimation. Kalman filters or linear quadratic estimation as they are also known as, use measurements/observed values of a variable of interest (the state variable) with the goal of making an inference about it. They work in a two step process. Namely, in the first step, the prediction step, the Kalman filter produces an estimate of the current state, along with its probabil- ity distribution. Once the outcome of the next measurement is observed, the previously produced estimates are updated. It is a recursive procedure, which means that it only needs the present observations and the previously calculated state and its uncertainty matrix, to estimate the current state variable. The latter hands them the advantage of running in-real time.

However, Kalman filters are linear model-based estimators, which means that they assume linearity of the underlying dynamical system (Meinhold \& Singpurwalla, 1983). In order to overcome this and to address the non-linearities in either the process model or the observation model or both, there exist the EKF and the UKF. The former assumes that the nonlinear functions are differentiable and linearizes about an estimate of the current mean and covariance while the latter uses deterministic sampling to form a new mean and covariance estimate (Tahan, Tsoutsanis, Muhammad, \& Abdul Karim, 2017) with a sampling technique known as the unscented transform (UT) to determine a minimal set of sample points (sigma points) around the mean.

The most popular model-based method is particle filters (Chen Xiongzi, Yu Jinsong, Tang Diyin, \& Wang Yingxun, 2011). PF method is a Sequential Monte Carlo (SMC) technique for implementing a recursive Bayesian filter using Monte Carlo simulations. SMC methods are a set of simulation-based techniques that provide an interesting approach to compute the posterior distributions of states. They approximate the optimal filtering by representing the probability density function with a population of particles, which are simply random samples (Daroogheh, Meskin, \& Khorasani, 2013). The basic idea is to develop a non-parametric representation of the system state probability density function in the form of a set of particles with associated importance weights. The particles are sampled values from the unknown state space and the weights are the corresponding discrete probability masses. As the filter then iterates, the particles are propagated according to the system state transition model, while their weights are updated based upon the likelihoods of the measurement given the particle values. They are a powerful and effective tool for accomplishing state and parameter estimation and allow for prediction in nonlinear dynamical systems where the noise in the observations comes from an arbitrary distribution and not just Gaussian. For more details regarding PF, we refer the interested reader to (Arulampalam, Maskell, Gordon, \& Clapp, 2002).

\subsection{Data-driven Approaches}

As opposed to, a data-driven method is much easier to be developed and applied in practical applications and is the recommended technique when the feasibility study implies a difficulty in obtaining a physical model. The low cost of algorithm development and little knowledge required about physics of the studied system, makes this approach preferable 
by prognostics system developers (Zhao, Liang, Wang, \& Lu, 2017). In addition, data-driven methods provide a high reliability and an effective computation, in spite of system complexity. Data-driven approaches mainly rely on techniques in the field of Artificial Intelligence (AI), which has many ready-to-use tools that could be applied directly with minor modifications. Nonetheless, compared to the physics-based method, the data-driven method requires a large amount of data, including both historical observation and current condition monitoring data. In principle, the more failure events are included in the data, the higher the accuracy of the estimation obtained. However, failure events are normally rare in any industry. In addition, this data is expensive and its accessibility is strictly limited for many reasons. Data-driven approaches for PHM can be classified as falling within one of the following two classes; i) statistical approach, and ii) machine learning approach.

The statistical approach uses statistical parameters, such as mean, variance, median and so on, to make predictions based on known or unknown underlying probabilistic distributions. Statistical approaches are generally considered to be simple if the underlying statistical property (i.e. probability distribution) is known. This type of approach is called parametric approach. Statistical parameter estimation techniques and hypothesis testing can be applied in this case to detect the presence of anomalies in the data. Here we list some examples of the statistical approaches. These include; hypothesis testing, analysis of variance (ANOVA), maximum-likelihood (ML) estimation, Gaussian mixture modelling (GMM), WilcoxonMann-Whitney test, Bayesian networks (BN), hidden Markov model (HMM), and principal component analysis (PCA).

However, machine learning approaches make predictions based on acquired data (such as healthy and failure data) by converting the gathered data into useful information which can be used in conjunction with sensor data to provide future predictions. Here we list some examples of the machine learning approaches. These include; nearest neighbour $(\mathrm{NN})$, neural networks, support vector machine (SVM), decision tree, random forest, etc. Readers who are interested more in the data-driven approaches can find more details in review papers on the data-driven approach and algorithms for PHM (Sikorska, Hodkiewicz, \& Ma, 2011a; Tsui et al., 2015; Sutharssan et al., 2015).

\subsection{Hybrid Approaches}

As previously mentioned, both model-based and data-driven prognostics approaches have their own merits and limitations. The hybrid (or fusion) prognostics approach, which is a newly developing approach, aims to integrate the merits of these different approaches while minimizing limitations for better system and/or component level health state estimation and RUL prediction. It is a promising method because it can compen- sate the lack of knowledge about the system's physics and the lack of data (Alia, Chebel-Morello, Saidi, Malinowski, \& Fnaiech, 2015; He, Williard, Chen, \& Pecht, 2014a; Baraldi, Compare, Sauco, \& Zio, 2013). This fusion can be performed either before the RUL estimation which is called preestimate, or after the RUL estimation to obtain the final RUL which is called post-estimate.

We, in this section, provided a brief overview about the PHM approaches. Each approach has its own merits and limitations. For practitioners, to select and implement a PHM approach is based on the application, the available data and their knowledge about the monitored system. Case studies and applications of each approach will be reviewed separately for automotive and aerospace industries.

\section{Performance metrics}

An important step in the successful deployment of a PHM system is prognosis certification (Saxena et al., 2008). However, the community lacks on a standardized approach to compare different methods in order for someone to identify the most suitable algorithm among a variety of possible choices. Additionally, there is an absence of a common ground, that is, benchmark datasets or models on which the techniques can be fairly compared. Performance metrics allow for the evaluation of different algorithms which can be tested rigorously and evaluated by different measures before they can be certified and thus employed in a real-world application. Furthermore, the existence of metrics, allows for establishing design requirements, specifications, guidelines or characteristics that can be used consistently to ensure that methods are fit for their purposes (Saxena et al., 2008), and moreover is important for scientific, administrative and economic reasons (Brier G.W. \& Allen R.A., 1951). From a scientific perspective, they matter due to the fact that they provide performance evaluations and therefore an objective way to discern how prognostic models affect the quality of the prediction. This thorough understanding yields valuable knowledge and can guide research and development efforts in the right direction. This refinement of the methods can result in better performance scores justifying the investment in PHM in areas that have not picked it up yet, as well as estimating the return-on-investment (ROI).

\subsection{Prognostics Metrics}

Prognostics metrics can be classified into three broad categories, based on the end-use of prognostics information. These are: algorithm performance metrics, computational performance metrics and cost-benefit metrics. Since this review paper is covering algorithmic methods in PHM, we will briefly describe the two latter categories before moving on to the former.

Cost-benefit metrics, such as life cycle cost, ROI, technical 
and total value, intend to measure the benefit gained by using prognostics. Table 3 in (Saxena et al., 2008) has a thorough list of these metrics. Computational metrics on the other hand, assess the computational performance of algorithms in terms of time and memory space. These metrics are particularly important in applications where there is need for a realtime processing of data to make safety-critical decisions and in embedded applications, such as on board computers of a car or aircraft, which have limited space available.

Algorithmic performance metrics are usually based on accuracy and precision, although algorithmic performance on robustness and trajectory of the RUL also exists. Table 2 in (Saxena et al., 2008) has a thorough list of these metrics. Here we will discuss the "new" metrics presented in the same paper, as these specifically cater to PHM requirements. To keep the discussion concise we present three of these. For a thorough reading we direct the interested reader to (Saxena, Celaya, Saha, Saha, \& Goebel, 2009) and to Table 4 in (Saxena et al., 2008).

\subsubsection{Prognostic Horizon (PH)}

An important question (requirement) to be asked when performing RUL predictions for PHM is "how far in advance is it enough to predict with a desired confidence in the predictions" (Saxena et al., 2009). The reason for this is of course that it is desired to seek a prediction which is reliable but also is enough time in advance before the actual end-of-life (EOL), so there is time for appropriate maintenance action. This leads to the Prognostic Horizon (PH) metric.

$\mathrm{PH}$ is defined as:

$$
P H=E O L-i
$$

where:

$i=\min \left\{j \mid(j \in \ell) \wedge\left(r_{*}-\mathrm{EOL} \cdot \alpha \leq r^{l}(j) \leq r_{*}+\mathrm{EOL} \cdot \alpha\right)\right\}$, $\alpha$ is the allowable error bound around true EoL and thus $i$ is the first time index, when predictions satisfy $\alpha$-bounds. Furthermore, $\ell$ is the set of time indexes when predictions are being made, $l$ is the $l^{\text {th }}$ unit under test (UUT), $r_{*}$ is the ground truth RUL, $r(j)$ is the predicted RUL at time $j$. The PH is declared as soon the corresponding predictions enter the band of desired accuracy and its range resides in $\left(t_{E O L}-t_{p}\right)$ and $\max \left\{0, t_{E O L}-t_{E O P}\right\}$, where EOP stands for end of prediction. For instance an error bound of $a=1 \%$ identifies when a given algorithm starts predictin estimates that fall within $1 \%$ of the actual EOL. The more an algorithm predicts whithin the desired accuracy scores the better its PH score is. As can be seen in Fig. 1, PH1 is more desirable than PH2.

\subsection{2. $\alpha-\lambda$ Performance}

Another important requirement is determining whether the prediction falls within specified limits at particular times, that is how well an algorithm performs when additional data

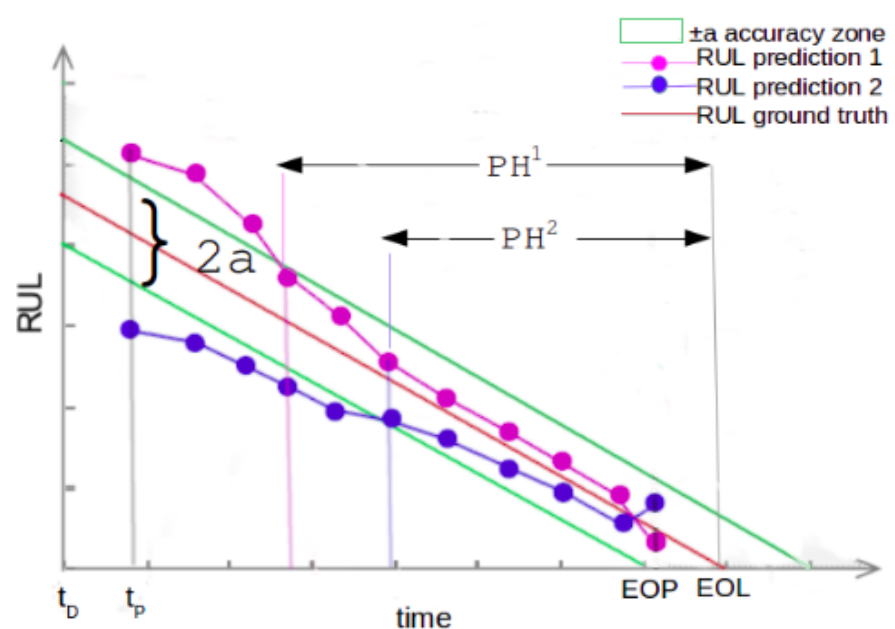

Figure 1. Prognostic Horizon. Adapted from (Saxena et al., 2010).

become available. Saxena et. al (Saxena et al., 2009) define $\alpha-\lambda$ accuracy, as the prediction accuracy to be within $\alpha * 100 \%$ of the actual RUL at a specific time instance $t_{\lambda}$. In words, this metric determines whether a prediction falls within $20 \%$ accuracy $(\alpha=0.2)$ halfway to failure from the time the first prediction is made $(\lambda=0.5)$. One needs to evaluate whether the following condition is met:

$$
(1-\alpha) * r_{*}(t) \leq r^{l}\left(t_{\lambda}\right) \leq(1+\alpha) * r_{*}(t)
$$

where $\alpha$ is the accuracy modifier, $\lambda$ is a time window modifier such that $t_{\lambda}=t_{P}+\lambda *\left(\mathrm{EOL}-t_{P}\right)$ and $t_{P}$ is the time at which the first prediction is made.

This metric is more rigid, compared to $\mathrm{PH}$, as it requires predictions to stay within a cone of accuracy. See Fig. 2 for the concept.

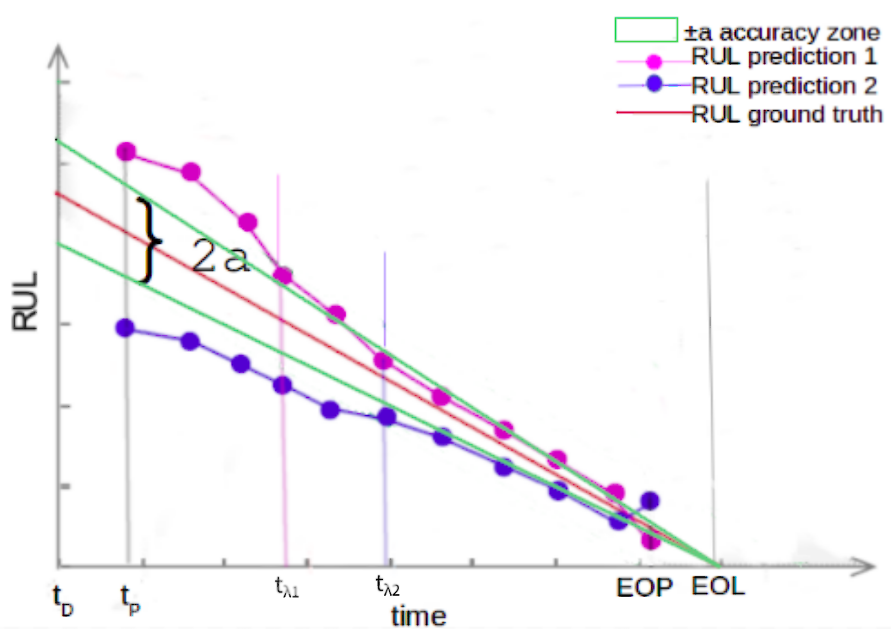

Figure 2. $\alpha-\lambda$ accuracy. Adapted from (Saxena et al., 2010). 


\subsubsection{Relative Accuracy (RA)}

Is similar to $\alpha-\lambda$ accuracy, but instead of finding out whether the predictions fall within given accuracy levels, at a time instants, it measures the accuracy levels. RA is defined as:

$$
R A_{\lambda}=1-\frac{\left|r_{*}\left(t_{\lambda}\right)-r^{l}\left(t_{\lambda}\right)\right|}{r_{*}\left(t_{\lambda}\right)}
$$

where $t_{P}$ is defined as before. See Fig. 3 for schematic representation. An algorithm with higher relative accuracy is desirable. In the figure we see two estimate curves (black and red) and the ground truth RUL (blue). We see that at time instant $t_{\lambda}$ the RA of the black estimate is (slightly) better than that of the rest. It is also visible that the RA of the red estimate decreases after time instant $t_{\lambda}$, while that of the black one increases.

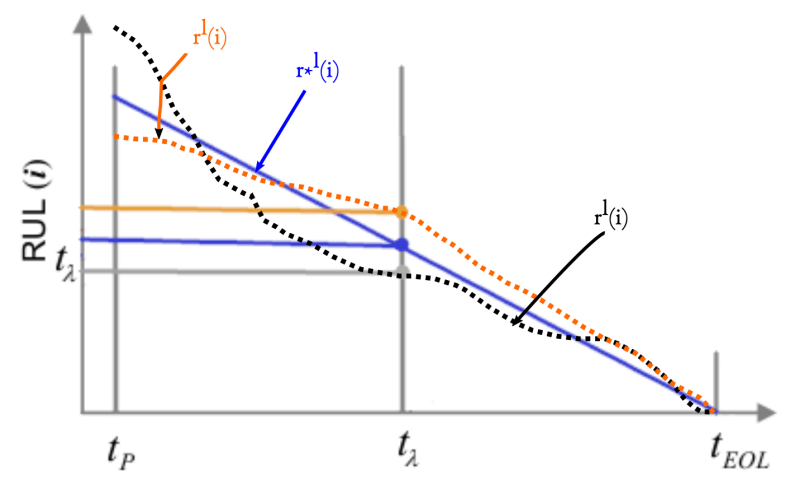

Figure 3. Schematic representation of RA. Adapted from (Saxena et al., 2009).

\subsection{Uncertainties in Prognostics}

To conclude this brief section, we must comment on uncertainty representation and management (URM) as this is an indispensable part of PHM. Accounting for uncertainties is of paramount significance in prognostics. Uncertainties arise from various sources such as: modeling uncertainties, measurement uncertainties, operating environment uncertainties, future load uncertainties, input data uncertainties. Such information is crucial for any prognostic estimate, otherwise it is of limited use and cannot be incorporated in mission critical applications. The reason for this is that the single point estimates that we described assume a deterministic algorithm or additional reasoning. Due to all the sources of uncertainty though, it is crucial that there is a confidence around the prediction. There are numerous ways for this, like probability distributions of the RUL instead of a single-point RUL estimate. In (Saxena et al., 2010) and (Saxena et al., 2009), in a very concise and detailed manner discuss the uncertainty issues and propose solutions by modifying PHM metrics and recommends suitable ways of graphically representing these metrics.

To summarize, we briefly discussed the motivation and the need behind performance metrics in PHM, by pointing out the shortcomings and presenting certain proposed methods. We described three of these methods, as we think they are very representative and briefly discussed the need of incorporating uncertainty representation as uncertainty is inherent in prognostics. Finally, it must be noted that the described metrics are intended for offline evaluation of prognostics and are not applicable for online cases. The reason for this is that PHM performance evaluation is an acausal problem that requires inputs from the events that are expected to take place in the future. The reason is that one needs to know the true EOL of the system to evaluate the prediction accuracy. Online evaluation will have to use methods to deal with uncertainties associated with future operating conditions in particular (Saxena et al., 2009),(Saxena et al., 2010). This requires future research in uncertainty representation.

So far we have covered PHM from a general perspective, introducing its significance, goal, methods, and shortcomings. In the remainder of this paper we will review PHM applications in the automobile and aerospace industries.

\section{PHM IN THE AUTOMOTIVE INDUSTRY}

According to a report in September 2003 published by the Commission of the European Community, repair and maintenance accounts for $40 \%$ of the total lifetime costs of vehicle ownership (Taie et al., 2012). In 2010, Toyota recalled more than 20 million vehicles due to technical issues, and nowadays software issues related to automotive controls account for an increasingly large percentage of the overall vehicles recalled. Therefore, a robust PHM system for automotive industry is required to overcome these issues. Recent advances in sensor technology, remote communication and computational capabilities, and standardized hardware/software interfaces are creating a dramatic shift in the way the health of vehicles is monitored and managed. Concomitantly, there is an increasing trend towards the forecasting of system degradation through a prognostic process to fulfill the needs of customers demanding high vehicle availability (Sankavaram et al., 2009).

\subsection{Classification of Automotive Sensors}

Prognostics and health management generally combines sensing and interpretation of environmental, operational, and performance - related parameters to assess the health of a product and predict RUL. Vehicles have very complex mechatronic structures consisting of systems and subsystems. Normally any subsystem comprises electromechanical processes, actuators, and sensors (Jeong et al., 2017). The sensors and actuators are associated and controlled with an engine control unit (ECU) which manages and screens the procedure. 
Knowing the types, functions and applications of sensors is required to develop a PHM system.

Due to rapid development of technology both the number and type of sensors keep increasing. However, on the basic level, the primary sensors in use today in automotive systems are reviewed and classified according to their three major areas of automotive systems application. They are powertrain, chassis, and body (Fleming, 2001). The powertrain encompasses every component that converts the engine's power into movement. This includes the engine, transmission, the driveshaft, differentials, axles; basically anything from the engine through to the rotating wheels. Area of systems application of the powertrain sensors are vehicle energy use, driveability, and vehicle performance. Chassis, also known as a vehicle frame, is the main supporting structure of a motor vehicle, to which all other components are attached. The chassis is considered to be the most significant component of an automobile. It is the most crucial element that gives strength and stability to the vehicle under different conditions. The main elements involved in chassis are steering, suspension (tire, springs, shock absorbers and linkages), vehicle breaking and stability. Area of systems application of the chassis sensors are mainly vehicle handling and safety. Anything that is not powertrain or chassis is included as a body systems application. It contains main elements such as occupant safety, security, comfort, convenience and information. The main sensors used in powertrain and chassis applications are listed in Table 1 and Table 2, respectively.

Table 1. Sensors used in powertrain application

\begin{tabular}{|l|l|}
\hline Functions & Powertrain sensors \\
\hline \hline Cylinder & $\begin{array}{l}\text { Pressure, combustion-gas ion cur- } \\
\text { rent }\end{array}$ \\
\hline Manifold & Pressure, temperature \\
\hline Turbo boost & Pressure \\
\hline Engine knock & $\begin{array}{l}\text { Vibration, combustion-gas ion cur- } \\
\text { rent }\end{array}$ \\
\hline Air intake & Mass flow, volume flow rate \\
\hline Engine torque & $\begin{array}{l}\text { Magnetostrictive, cylinder-ciring- } \\
\text { induced, crankshaft speed modula- } \\
\text { tion }\end{array}$ \\
\hline Camkshaft & Rotational motion \\
\hline Throttle, pedal & Rotary motion \\
\hline Fuel injection & Pressure \\
\hline Exhaust/catalyst & Temperature, catalytic activity \\
\hline Engine oil & $\begin{array}{l}\text { Pressure, level, quality (predic- } \\
\text { tive, ac-dielectric constant, cyclic } \\
\text { Voltammogram, thermal conductiv- } \\
\text { ity) }\end{array}$ \\
\hline Coolant system & $\begin{array}{l}\text { Temperature, level } \\
\text { Fevel, evaporation leak pressure, } \\
\text { flexible fuel composition }\end{array}$ \\
\hline Transmission & $\begin{array}{l}\text { Gearshift position, input/output } \\
\text { shaft speeds, temperature, pressure, } \\
\text { torque. }\end{array}$ \\
\hline
\end{tabular}

Table 2. Sensors used in chassis application

\begin{tabular}{|c|c|}
\hline Functions & Chassis sensors \\
\hline$\overline{\text { Brake System }}$ & Pressure, fluid level \\
\hline $\begin{array}{l}\text { ABS anti-lock } \\
\text { braking }\end{array}$ & $\begin{array}{l}\text { Wheel speed, pressure, lateral ac- } \\
\text { celeration }\end{array}$ \\
\hline Brake-by-wire & Pedal force/depression angle \\
\hline $\begin{array}{ll}\text { Electric } & \text { power } \\
\text { steering }\end{array}$ & $\begin{array}{l}\text { steering wheel angle, steering } \\
\text { wheel torque }\end{array}$ \\
\hline Vehicle stability & $\begin{array}{l}\text { Wheel speed, lateral acceleration, } \\
\text { yaw angular rate, steering wheel an- } \\
\text { gle }\end{array}$ \\
\hline $\begin{array}{l}\text { Active suspen- } \\
\text { sion }\end{array}$ & $\begin{array}{l}\text { Strut displacement, chassis height, } \\
\text { body acceleration (vertical, lateral, } \\
\text { longitudinal), yaw angular rate, roll } \\
\text { angular rate, steering wheel angle }\end{array}$ \\
\hline Tire pressure & $\begin{array}{l}\text { Wheel-to-wheel variance of rolling } \\
\text { speed, on-wheel sensor, wireless }\end{array}$ \\
\hline Tire temperature & On-wheel sensor, wireless \\
\hline
\end{tabular}

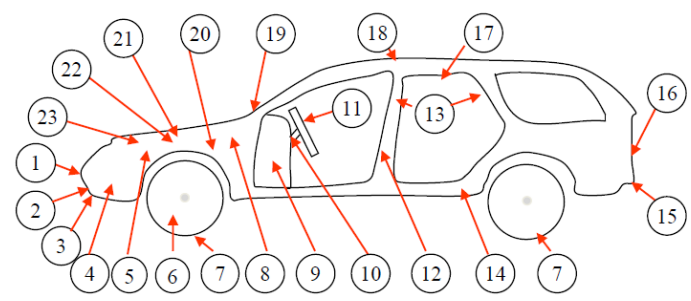

\begin{tabular}{|c|l|c|l|}
\hline No. & Monitoring and sensing & No. & Monitoring and sensing \\
\hline \multirow{2}{*}{1} & $\begin{array}{l}\text { Collision avoidance, night vision, and front crash } \\
\text { detection, forward obstacle sensor }\end{array}$ & 13 & Side airbag deployment sensor \\
\cline { 2 - 4 } & 14 & Angular acceleration (suspension) \\
\hline 2 & Vehicle distance sensor & 15 & Emissions sensor \\
\hline 3 & Road condition sensor & \multirow{2}{*}{16} & $\begin{array}{l}\text { Back-up collision, rear vision camera, } \\
\text { rear radar, rear obstacle sensor }\end{array}$ \\
\hline 4 & Side obstacle sensor & \multirow{2}{*}{17} & $\begin{array}{l}\text { Temperature and humidity sensor, and } \\
\text { comfort control }\end{array}$ \\
\hline 5 & Oil/fuel pressure and flow monitoring & 18 & Rollover detection \\
\hline 6 & Speed sensors & 19 & Rain sensor and wiper control \\
\hline 7 & Tire pressure monitoring & 20 & Power train control module \\
\hline 8 & Fire detection sensor & 21 & Throttle position monitoring and control \\
\hline 9 & Driver monitoring sensor & 22 & Battery monitoring \\
\hline 10 & Steering angle sensor and stability control & 23 & Ignition and engine control monitoring \\
\hline 11 & Yaw and acceleration sensors for airbag deployment \\
\hline 12 & Side crash detection & & \\
\hline
\end{tabular}

Figure 4. Important sensors of an automobile (Cheng et al., 2010).

If we classify automotive sensors according to their functions, we can do it as follows (Fleming, 2001): there are six types of rotational motion sensors, four types of pressure sensors, five types of position sensors, and three types of temperature sensors, two types of mass air flow sensors, five types of exhaust gas oxygen sensors, one type of engine knock sensor, four types of linear acceleration sensors, four types of angular-rate sensors, four types of occupant comfort/convenience sensors, two types of near-distance obstacle detection sensors, four types of far-distance obstacle detection sensors, and ten types of emerging. Fig. 4 shows important sensors and their positions on an automobile (Cheng, Azarian, \& Pecht, 2010). 


\subsection{Automobile: From Diagnostics towards Prognostics}

A diagnostic protocol normally communicates with Electronic Control Units (ECUs) and offers the application layer services from reading out Diagnostic Trouble Codes (DTC). The three well known diagnostic protocols are On-Board Diagnostics II (OBD-II), Unified Diagnostic Services (UDS), and Remote Diagnosis and Maintenance Systems (RDMS) (Taie et al., 2012). OBD-II refers to a vehicle's selfdiagnostic and reporting capability. OBD systems give the vehicle owner or a repair technician access to state of health information for various vehicle sub-systems. UDS defines the application layer, data link layer and the physical layer of the diagnostic communication. It does not specify all details, but some are left out to the manufacturers. Remote Diagnosis and Maintenance Systems (RDMS) is developed thanks to recent advances in remote communications, innovative mobile applications, human-machine interfaces, modelbased diagnostics, electronics and embedded system technologies. RDMS improves diagnostics methods and equipment in order to accurately locate and diagnose any malfunctions. Service technicians do not have to merely rely on visual and physical inspections alone to resolve vehicle problems. Moreover, these advances equip the automobiles with the capability to share in-vehicle sensor and diagnostic information with remote computers, enabling vehicle diagnosis and maintenance be performed remotely while the vehicle is being driven. They provide manufacturer specific repair information according to the problems identified by the Off/On-Board-Diagnosis systems. In addition, vehicle parameters can be monitored while the vehicle is being driven to determine when maintenance is necessary.

There is a trend towards the forecasting of system degradation through a prognostic process to fulfill the needs of customers demanding high vehicle availability. In 2012, Taie and co-authors (Taie et al., 2012) presented a novel automotive Remote Diagnosis Prognosis and Maintenance system (RD\&M). The elements of the proposed system include vehicles, smart phones, maintenance service centers, vehicle manufacturer, RD\&M experts, RD\&M service centers, logistics carry centers, and emergency centers. The system promotes the role of smart phones used to run prognosis and diagnosis tools based on Least Squares Support Vector Machine (LSSVM) multiple classifiers. During the prognosis phase, the smart phone stores the history of any forecasted failures and sends them, only if any failure already occurred during the diagnosis, to the RD\&M service center. The latter will then forward it to RD\&M experts as a real failure data to improve the training data used in prognosis classification and predication of the remaining useful life (RUL).

Classifying health status of the automatic gearbox was a case study for this RD\&M system. In this case study, the training data was provided by the original equipment man- ufacturer (OEM) system experts. Based on the relation between tachometer readings, vehicle speed readings and gearbox temperature reading, the gearboxes are classified into four classes such as "OK", "RUL 40\%", "RUL 10\%" and "NOK". The gearbox is considered normal (OK) if the gearbox temperature is normal and the gear ratio (ratio between vehicle speed and motor speed) is within acceptable range, on the other hand, failure (NOK) is detected if the gearbox temperature was above normal regardless the values of the gear ratio, finally there were two classes of warnings (RUL $40 \%$ and RUL 10\%) where the RUL was depending on the gear ratio. The training was done on 100 examples of the above mentioned three sensor readings. Cross validation was done using leave one out technique to evaluate the classification of LS-SVM versus the classical K-nearest neighbor K-NN. The accuracies were 0.93 and 0.82 for LS-SVM and K-NN, respectively.

Very recently, in 2017, Shafi and co-authors (Shafi, Safi, Shahid, Ziauddin, \& Saleem, 2018) developed a platform for fault prediction of four main subsystems of vehicles: fuel system, ignition system, exhaust system, and cooling system. It is called 'Vehicle Remote Health Monitoring and Prognostic Maintenance System (VMMS)'. In the VMMS, sensor data is collected when the vehicle is on the move, both in faulty condition (when any failure in specific system has occurred) and in normal condition. The data is transmitted to the server which analyzes the data. Interesting patterns are learned using four classifiers such as Decision Tree, Support Vector Machine, K-Nearest Neighbor, and Random Forest. These patterns are later used to detect future failures in other vehicles which show the similar behavior. The approach is developed with the goal of expanding vehicle up-time and was demonstrated on 70 vehicles of Toyota Corolla type. Accuracy comparison of all classifiers is performed on the basis of Receiver Operating Characteristics (ROC) curves.

VMMS has three main layers including data generation, data processing and remote monitoring. In the first layer, an OBD II scanner is connected with the vehicle through OBD II port. This scanner behaves like a bridge between vehicle and a portable device, such as a mobile or a laptop which supports Bluetooth. All the sensor's data in form of Diagnostic Trouble Codes (DTC) is generated when the vehicle is on the move and sent to the portable device. In the data processing layer, the first step is feature selection in which the data stream of DTC is filtered in a feature selection process using the expert's suggestions. Then a Principle Component Analysis (PCA) is applied on the data set for feature reduction. After that four classification algorithms are used in the classification phase including Decision Tree, Random Forest, K-NN, and SVM. Then, results are stored on the server for further derivation which is used for fault prediction and remote monitoring of the vehicle. In the remote monitoring layer, the owner or concerned person of the vehicle can monitor the cur- 
rent condition of the vehicle remotely like fuel status, speed, and current position. The driver or the owner of the vehicle is notified about the failure of any subsystem of the vehicle through automatic notification.

The VMMS was experimentally evaluated on the four main systems of 70 vehicles of Toyota Corolla type including ignition -, exhaust -, fuel -, and cooling. A stream of DTCs was produced by sensors with a sampling frequency of $1 \mathrm{~Hz}$ when the vehicle was on the move for each system under experiment. Each reading is taken as an example and contains around 20 DTCs. The dataset consists of 150 examples. DTC generated by sensors is considered as an attribute or feature. The feature value is set to 1 if that particular DTC is generated and set to 0 otherwise. The output of the system or class label is also in binary form. If the system is in operation then the output is set to 0 which means that the vehicle is in a safe condition. If a fault occurred or the system breaks down, then the output class label is set to 1 . So the generated dataset is completely binary in nature. That's why the authors selected Decision Tree, Random Forest, K-NN, and SVM algorithms for classification as they perform well on binary data. All classifiers are evaluated with 10 -fold cross validation.

The performance of each algorithm on a particular subsystem is evaluated on the basis of accuracy, precision, recall, and F1 score measures. Here accuracy is the percentage of the total number of predictions that was found correct. Precision is true positive accuracy. Recall is true positive rate. Lastly, the F1 score is an accuracy indicator which is measured using the precision and the recall. The precision, recall, and F1 score are calculated as follows:

$$
\begin{gathered}
\text { Precision }=\frac{T P}{(T P+F P)} \\
\text { Recall }=\frac{T P}{(T P+F N)} \\
F 1=\frac{2 * \text { Precision } * \text { Recall }}{(\text { Precision }+ \text { Recall })}
\end{gathered}
$$

where TP, FP, and FN are true positive, false positive and false negative, respectively. The percentage accuracy of all algorithms for all subsystems is shown in Table 3. In all cases, results show that the performance of all algorithms is very good and SVM is the best classifier. The lowest accuracy of the SVM model is $96.6 \%$ which is achieved on the ignitionand cooling systems, while the best accuracy is $98.5 \%$ which is achieved on the fuel system.

RDMS and VMMS perform very well on the fault- detection and classification. However, forecasting the RUL which is the heart of any PHM system is still missing. There has not been yet a robust PHM platform which can be used for prognostics of the entirely vehicle. We are exploring our review on prognostics for subsystems and components.

Table 3. Accuracy of the VMMS performance on the main four subsystems

\begin{tabular}{|l||l|l|l|l|}
\hline Classifiers & Ignition & Fuel & Exhaust & Cooling \\
\hline \hline DT & 72.5 & 76.5 & 78.5 & 75.9 \\
\hline SVM & 96.6 & 98.5 & 98.0 & 96.6 \\
\hline K-NN & 81.9 & 94.6 & 89.9 & 94.6 \\
\hline RF & 79.2 & 90.0 & 88.6 & 89.3 \\
\hline
\end{tabular}

\subsection{PHM for Battery}

Batteries are a core component of many machines and are critical to the system's functional capabilities. Battery failure could lead to reduced performance, operational impairment, and even catastrophic failure, especially in aerospace and automobile systems (Goebel, Saha, Saxena, Celaya, \& Christophersen, 2008). Additionally, in terms of air pollution, greenhouse gas emissions, and economy, using electric vehicles is nowadays preferred by many people. Significant work has been done to determine the states and conditions of batteries. Readers who are particularly interested in this topic can find more details in these review articles: Zhang et al. (J. Zhang \& Lee, 2011), Rezvanizaniani et al. (Rezvanizaniani, Liu, Chen, \& Lee, 2014), Berecibar (Berecibar et al., 2016), and Lipu et al. (Lipu et al., 2018) and recent publications: You et al. (You, Park, \& Oh, 2016), Dang et al. (Dang et al., 2016), Yang et al. (F. Yang, Xing, Wang, \& Tsui, 2016), Ye et al. (Ye, Guo, \& Cao, 2017), Jafari et al. (Jafari, Khan, \& Gauchia, 2018), Tian et al. (Tian, Xiong, \& Yu, 2019), Razavi et al. (Razavi-Far, Chakrabarti, Saif, \& Zio, 2019), and Downey et al. (Downey, Lui, Hu, Laflamme, \& Hu, 2019). In short, the main tasks of PHM for battery industry are state-of-charge (SOC) estimation, current/voltage estimation, capacity estimation and remaining-useful-life (RUL) prediction. There exist many estimation methods including model-based, data-driven and hybrid. Some examples of the model-based methods are open-circuit voltage, current integral, internal resistance measurement, impedance measurement, (discrete) Thevenin model, Coulomb counting, Particle filter, and (adaptive extended) Kalman filter, etc. Commonly used data-driven methods are ANN, SVM, RVM, Auto - regressive moving average, and Fuzzy logic. Usually, the model-based estimations have less computational cost and high time efficiency. However, these methods perform well only on absolutely clean and precise data. In reality, data can contain uncertainties and noise. Data-driven methods perform better with such type of data. Nonetheless, these methods exhibit complex computation and need a large amount of data for appropriate training.

Hybrid methods, therefore, have been developed to overcome the limitations and thus improve the prediction performance. 
We here highlight some outstanding examples.

In 2012, Liu et al. (J. Liu, Wang, Ma, Yang, \& Yang, 2012) developed a fusion prognostic framework to improve the accuracy of system state long-horizon forecasting. This framework strategically integrates the strengths of the datadriven prognostic method and the model-based particle filtering approach in system state prediction while alleviating their limitations. In the proposed methodology, particle filtering is used for system state estimation whereas a data-driven method is used to predict future measurements for the modelbased method. The predicted measurements from the datadriven method can be regarded as new measurements in the model-based method when there is a lack of measurements during long-term prediction. As an application example, the developed fusion prognostic framework is employed to predict the RUL of lithium ion batteries through electrochemical impedance spectroscopy tests. The experimental results demonstrate that the proposed fusion prognostic framework is an effective forecasting tool that can integrate the strengths of both the data-driven method and the particle filtering approach to achieve more accurate state forecasting.

In 2013, Xing et al. (Xing, Ma, Tsui, \& Pecht, 2013) developed an ensemble model to characterize the capacity degradation and predict the remaining useful performance (RUP) of lithium-ion batteries. Their model fuses an empirical exponential and a polynomial regression model to track the battery's degradation trend over its cycle life based on experimental data analysis. Model parameters are adjusted online using a particle filtering (PF) approach. Experiments were conducted to compare the ensemble model's prediction performance with the individual results of the exponential and polynomial models. The ensemble model demonstrated better prediction performance (smaller prediction errors and a narrower standard deviation). This is because this model balanced the global and local regression performance. The developed model was evaluated on two different battery sets with two different rated capacities. For both kinds of battery samples, credible and reliable prediction results were achieved. However, there are some limitations in applying this developed model. Firstly, temperature effect is not considered in model. Secondly, in some cases, it is difficult to quantify the actual maximum capacity because the battery is usually not fully discharged in every cycle. The authors suggested to map the capacity of the partial discharge into the equivalent fully discharged capacity before using the developed model. The transform relation can then be explored by measuring the different voltages and finding the interaction between the random cut-off discharge and fully discharged voltage.

In the same year, Wang et al. (D. Wang, Miao, \& Pecht, 2013) developed a capacity prognostic method to estimate the RUL of lithium-ion batteries. This method consists of a relevance vector machine and a conditional three-parameter capacity degradation model. The aim of the relevance vector machine is to find a few representative basis functions to derive the prediction model by using sparse Bayesian learning. The conditional three-parameter capacity degradation model is used to fit the predictive values at the cycles of the relevance vectors. Extrapolation of the conditional three-parameter capacity degradation model to a failure threshold is used to estimate the RUL of lithium-ion batteries. To illustrate how the developed battery capacity prognostic method can be used, three instance studies for batteries A1, A2 and A3 were conducted. The results showed that the developed method was able to predict the future health condition of lithium-ion batteries. They found that as more capacity degradation data is used to train the relevance vector machine, the accuracy of the battery RUL prediction is improved.

In 2014, He et al. (He, Williard, Chen, \& Pecht, 2014b) developed an artificial neural network-based battery model to estimate the SOC, based on the measured current and voltage. This model uses unscented Kalman filter (UKF) to reduce the errors in the neural network-based SOC estimation. The method was validated using $\mathrm{LiFePO}_{4}$ battery data collected from the Federal Driving Schedule (FDS) ${ }^{3}$ and dynamical stress testing. This UKF-based approach was implemented to filter out the errors in the neural network estimation. They reported the root mean squared (RMS) errors of the SOC estimation were within $2.5 \%$ to $3.5 \%$ for different temperatures. There are three main contributions of this study namely i) a constructive searching approach was developed to find the optimal neural network structure for SOC estimation, and ii) a state-space model was developed that combines coulomb counting and neural networks. Moreover, a UKF approach was implemented to improve the neural network SOC estimation under different temperatures. The developed method eliminates the need to determine an open circuit voltage SOC lookup table, unlike equivalent circuit model-based SOC estimation. The field collected data can be used to update the neural network and increase the estimation accuracy. iii) This method does not rely on the physics of batteries, since a neural network is a data-driven approach. As a result, the developed approach can be readily applied to batteries with different chemistries.

In 2015, Zheng and Fang (Zheng \& Fang, 2015) developed a method that uses UKF with relevance vector regression (RVR) to predict RUL of short-term capacity of batteries. A RVR model is employed as a nonlinear time-series prediction model to predict the UKF future residuals which otherwise remain zero during the prediction period. The objective of the integrated UKF-RVR is to predict the battery RUL in a way that the battery model parameters can be continuously and recursively updated by properly incorporating prediction

\footnotetext{
${ }^{3}$ https://www.epa.gov/emission-standards-reference-guide/epa-us06-orsupplemental-federal-test-procedure-sftp
} 
information from the RVR method and the last true residual value through model-based UKF method. The performance of the proposed method was validated and compared to other predictors with the experimental data of four batteries. Authors claimed that, according to the experimental and analysis results, the proposed approach exhibits high reliability and prediction accuracy, which can be applied to battery monitoring and prognostics, as well as be generalized to other prognostic applications.

\subsection{PHM for Suspension}

Suspension is the system of tires, tire air, springs, shock absorbers and linkages that connects a vehicle to its wheels and allows relative motion between the two. Suspension systems must support both road-holding/handling and ride quality, which are at odds with each other. The tuning of suspensions involves finding the right compromise. It is important for the suspension to keep the road wheel in contact with the surface as much as possible, because all the road or ground forces acting on the vehicle do so through the contact patches of the tires. The suspension also protects the vehicle itself and any cargo or luggage from damage and wear. The design of front and rear suspension of a car may be different. In general, suspension systems can be broadly classified into three subgroups: dependent, independent and semi-independent suspensions (Dishant, 2017). These terms refer to the ability of opposite wheels to move independently of each other. A dependent suspension normally has a beam or driven live axle that holds wheels parallel to each other and perpendicular to the axle. This type of suspension system acts as a solid link between two wheels such that any movement of one wheel is translated to the other wheel. Also, the force is translated from one wheel to the other. In contrast, an independent suspension allows wheels to rise and fall on their own without affecting the opposite wheel. This is a widely used suspension system in passenger cars and luxury cars due to its advantages over a dependent suspension system.

Springs are the main component in the suspension system which help to reduce road shocks and vibration of a vehicle. Depending on vehicles different types of springs are used and they can be classified as: leaf spring, helical/coil spring, torsion bar, rubber spring, or hydro-pneumatic spring. Springs that are too hard or too soft cause the suspension to become ineffective because they fail to properly isolate the vehicle from the road. Vehicles that commonly experience suspension loads heavier than normal have heavy or hard springs with a spring rate close to the upper limit for that the vehicle's weight. This allows the vehicle to perform properly under a heavy load when control is limited by the inertia of the load. Riding in an empty truck used for carrying loads can be uncomfortable for passengers because of its high spring rate relative to the weight of the vehicle. A race car would also be described as having heavy springs and would also be uncom- fortably bumpy. A luxury car, taxi, or passenger bus would be described as having soft springs. Vehicles with worn out or damaged springs ride lower to the ground, which reduces the overall amount of compression available to the suspension and increases the amount of body lean. Performance vehicles can sometimes have spring rate requirements other than vehicle weight and load.

Having a good maintenance scheduling for the suspension system supports vehicle's comfort and safety. Common mechanisms that lead to suspension failure are crack propagation, corrosions, chloride attack, creep, excessive deformation and deflection, damage accumulation, and fatigue damage. Luo et al. (J. Luo, Pattipati, Qiao, \& Chigusa, 2008) and Jaoude et al. (Jaoude, 2015) focused on fatigue analysis to predict the RUL of springs. They used real physical principles/laws to establish their prediction models. These physical principles include the stress-cycle curve, Rainflow model (Matsuishi \& Endo, 1968), Paris-Erdogan's (Sobczyk $\&$ Spencer, 1993) and Palmgren-Miner's laws (Miner, 1945). In these papers, a systematic model-based prognostic process is presented to successfully predict the RUL of a system with multiple operational modes, load conditions, environmental conditions, and road conditions. However, the successful use of the prediction models is limited to the simulation data. The Application of the process to real-world suspension systems is still missing.

In 2017, Yang et al. (C. Yang, Song, \& Liu, 2017) used a data-driven method to predict the RUL of hydro-pneumatic springs. The main issues that cause failure in hydropneumatic springs are gas leakage and oil leakage. The authors developed a time domain fault feature method, based on degraded pressure under the same displacement condition, and a feature extraction method based on linear interpolation methods and redefined time intervals. They then combined this feature extraction method with a data-driven prognostic method that was based on support vector regression (SVR) to predict the failure probability and the RUL values of these systems. Real vehicle historical data and simulation data were used to verify the feasibility of the proposed method. In both cases, they found a good agreement between the predicted and the true values. However, the RUL could be predicted ahead only a few hours due to limitations of the available data. This could only help drivers to prevent bad accidents, but it is not very meaningful for maintenance scheduling.

In 2018, Luo et al.(H. Luo, Huang, \& Zhou, 2018) developed a health monitoring approach for a MacPherson strut suspension systems based on vibration signals. This approach can be considered as a hybrid model, because it uses the PalmgrenMiner rule and the Rainflow model to estimate the damage and a neural network to predict the partial damage level. This method consists of two major parts: multi-Gaussian fitting 
feature extraction and a long short-term memory (LSTM) based damage identification. After the application of a multiGaussian fitting method to extract meaningful and discriminative features, a proper LSTM neural network is built to predict the real-time partial damage level. Then the health status, in the form of remaining useful life, is estimated.

The performance of the proposed health monitoring method was experimentally verified by torsion beam suspension durability tests. To collect data sets, they conducted two major experiments. The first experiment was to specify the driving cycles that can reflect real operating conditions. The second one was to specify the measured signals. These aspects are addressed as follows: under different driving cycles, the suspension component bears different vibration load, which causes obvious partial damage change. Therefore, the partial damage level is mainly affected by driving cycles. To make the monitoring system work effectively under real operation conditions, driving cycles are selected according to the guideline of comprehensiveness and distinctiveness. In this work, bench and road tests under various driving cycles were implemented to simulate the real operation conditions. Bench tests were performed to obtain data sets for training the LSTM model. The measured signals in the data sets of road tests were used to validate the LSTM model. As it is of high cost and time consuming to generate data by road tests, they conducted bench tests to collect training data sets. For the case of measured signals, the vibration signals are considered as candidate inputs for the health monitoring system. This is because vibration is related to damage identification of the suspension component and it is available on the Controller Area Network (CAN) of a standard vehicle. For the sake of applicability of the monitoring system, candidate inputs are composed of signals collected by common sensors: the displacement and angular velocity of the vehicle body in the $\mathrm{x}, \mathrm{y}$ and $\mathrm{z}$ directions, the deformation of two springs and two shock absorbers in the rear torsion beam suspension, the deformation of the two springs in the MacPherson front suspension and the vertical acceleration of four spindles in the center of wheels. In both cases, they achieved striking prediction accuracy, while requiring low computation time.

\subsection{PHM for other Automotive Components}

In 2016, Sankavaram and co-authors established an inference-based prognostic framework for health management of automotive components (Sankavaram et al., 2016). The framework is called Cox-PHM. Cox-PHM uses datadriven methods to detect fault diagnosis and degraded state trajectories and to estimate the RUL of components. The framework takes into account the cross-subsystem fault propagation, a case prevalent in any networked and embedded system. The key idea is to use a Cox proportional hazards model to estimate the survival functions of error codes and symptoms (probabilistic test outcomes/prognostic indicators) from failure time data and static parameter data, and use them to infer the survival functions of components via a so-called soft dynamic multiple fault diagnosis algorithm. The average RUL can be estimated from these component survival functions. The proposed prognostic framework consists of two phases: an offline training and validation (model learning) phase, and an online testing (deployment) phase.

In the Cox-PHM, data is classified into three types namely i) archived failure data (Type I data): age (or a surrogate function such as the mileage or operational time) of the vehicle at the time of failure, i.e., age when an error code or symptom is observed, or a component is replaced; ii) static environmental and status parameter data (Type II data); and iii) dynamic data (Type III data): time-series data and periodic status data. The framework employs two key techniques: (i) Cox proportional hazards model (Cox-PHM) (Klabfleisch \& Prentice, 2002), and (ii) soft dynamic multiple fault diagnosis (soft DMFD) inference algorithm (Singh, Kodali, \& Pattipati, 2009). The Cox-PHM computes the survival functions of tests (or error codes), whereas the soft DMFD algorithm is used to infer failing components in coupled systems. The soft DMFD algorithm determines the most likely evolution of component states that best explains the observed soft test failure outcomes (i.e., complementary test survival probabilities).

The training phase consists of two steps. In Step 1, Type I and Type II data are used to compute static data-modulated survival functions for components, error codes, symptoms and any observable test outcomes via the Cox proportional hazards model. In the testing phase, when new feature data (Type III dynamic data) is obtained via online data acquisition systems, the survival probabilities of error codes are estimated using the Cox-PHM model as well as the baseline hazard functions obtained from the offline module (from Type I and Type II data). The RUL of a component at any time can be computed from the survival function by defining a threshold on the survival probability.

The framework was demonstrated on datasets derived from two automotive systems: i) a dataset derived from an automotive electronic throttle control (ETC) system simulator with failure time data, static parameter data, and simulated test outcomes; and ii) a dataset derived from an automotive regenerative braking system (RBS) with failure time data, and static as well as dynamic parameter data obtained from simulationbased fault injection experiments conducted in MATLAB / Simulink.

In the first application, the prognostic approach is applied to a dataset derived from an automotive ETC subsystem simulator. The function of an ETC subsystem is to determine the necessary throttle opening using sensors (such as the accelerator pedal position, the engine RPM, and the vehicle speed) and drive the actuator to obtain the required throttle posi- 
tion via a closed-loop control algorithm in the engine control module (ECM). The ECM also monitors the health of the engine subsystem by processing parameter identifier data (PIDs) collected from various sensors and generates diagnostic trouble codes (DTCs or error codes) when a failure occurs in any component. The dataset derived from the ETC simulator consisted of 11 error codes (DTCs), 479 status parameters (PIDs) collected at the time of DTC firing, accelerated age of the vehicle and the repair/replacement actions (i.e., repair codes (RCs)) performed on the system. A total of five different repair codes are present in the training data. That includes: RC1 (Accelerator Pedal Replacement), RC2 (Throttle Body Assembly Replacement), RC3 (Accelerator Pedal Position Sensor Replacement), RC4 (Powertrain Control Module Replacement) and RC5 (Throttle Position Sensor Replacement).

Under a single fault assumption, the authors showed the estimated component failure probability for all repair codes. The $R^{2}$ fits were quite high ( $>90 \%$ ) for the RC1, RC2, RC3 and RC5 codes. For the RC4 code, although the R-square fit was low $(78 \%)$, at the long age axis, the estimated failure probability was higher than the actual component fault probability. This suggests that the algorithm can estimate/predict the failing component before the actual component reaches the failing threshold - which is expected from an effective prognostic algorithm. In addition to RC4, the failure probability of RC2 was also significant compared to other repair codes. This was because RC2 was a hidden fault of RC4 and the algorithm inferred RC2 as failing component with failure probability of less than 0.5 .

In the second application, the prognostic framework was applied to estimate the sensor and parameter degradations in a regenerative braking system (RBS). The RBS consists of a driver model, a powertrain controller, component controllers, and the powertrain model. The driver model simulates the drive cycles by setting accelerator and brake pedal positions to achieve the desired vehicle speed. The output from this block is the driver's torque demand at the wheels; this acts as the input to the powertrain controller (PTC). The PTC is the supervisory controller making the high-level decisions that affect the general state of the powertrain, the operating mode of the vehicle, and accordingly deliver the torque requests to the component controller. Subsequently, the component controller converts these torque requests into component commands. These commands are, in turn, treated as the actuator commands by the individual components in the powertrain model to achieve the requested torque and, consequently, report the system status (e.g., engine speed, battery state of charge) to the supervisory controller. The powertrain model comprises of all the components that mimic the behavior of hardware components, such as the engine, the battery, and the motor.
There are 25 signals that were being monitored in the RBS system. Important signals contained: i) sensor signals, such as temperature, speed, and current measurements from the hardware components in the powertrain model; ii) motor, wheel, and engine torque demands sent from the powertrain controller to the component controllers; and iii) component commands sent from the individual ECUs to the hardware components in the powertrain model. To demonstrate the framework, two faults were considered such as motor speed sensor fault and wheel inertia fault. These faults were injected into the model as additive biases on the measured signals. In both cases, the estimated component degradations were in good agreement with the truth with an R-square fit of about 96\%. An estimation of RUL at any time could be obtained directly by defining a threshold on the failure probability.

To summarize, the authors presented a novel approach for fault prognosis problem in coupled systems by combining three types of data, i.e., failure time data, static environmental and status parameter data, and dynamic data. The framework employed the Cox PHM to infer the survival functions of components and subsequently estimated the component degradations via the soft dynamic multiple fault diagnosis algorithm. The framework are applied to two different automotive cases to infer the component degradations (complementary survival functions) and the inference algorithm estimated the component failure probabilities with a good $R^{2}$ fit. Additionally, the authors claimed that although the proposed framework was validated on automotive systems, it has the potential to be applicable to a wide variety of systems, ranging from aerospace systems to buildings to power grids. However, the framework does not include the uncertainty in RUL estimation. It is very important for maintenance scheduling.

A recent hybrid framework developed by Nguyen et al. (Nguyen, Limmer, Yang, Olhofer, \& Bäck, 2019) provides a method to generate RULs data with uncertainty of four essential components of a passenger car, namely engine, brake pads, springs, and tires. In this work, authors used CarMaker simulation to simulate a fleet of 200 cars with different driving scenarios in New York City. They then used physicsbased and data-driven approaches to predict the RUL of the above mentioned components. Their results show a good consistency of both approaches. This framework can be used for establishing an optimal maintenance schedule for a vehicle fleet (Wang, Limmer, Olhofer, Emmerich, \& Bäck, 2019), such as a fleet of a taxi company.

For other components, several frameworks were developed such as for engine (J. Wang, Mao, Zhu, Song, \& Zhuo, 2009; M.-H. Wang, Chao, Sung, \& Huang, 2010; Beatrice, Guido, Napolitano, Iorio, \& Giacomo, 2011; Ko et al., 2014; Ashok, Denis Ashok, \& Ramesh Kumar, 2016, 2017), antilock braking (J. Luo, Namburu, Pattipati, Qiao, \& Chigusa, 2010), (axle-) gearbox (X. Zhang, Kang, Zhao, \& Cao, 2013; Shao, 
Liang, Gu, Chen, \& Ball, 2011; Saimurugan, Praveenkumar, Sabhrish, Sachin Menon, \& Sanjiv, 2016), and steering system (Ming Yu \& Danwei Wang, 2014; Lin \& Ghoneim, 2016). Nonetheless, in all cases, the success is limited to diagnostic. The prognostic application, which is the most important part of PHM, is still either incomplete or missing. Establishing a PHM system for these components remains a big challenge.

\subsection{Discussions and Conclusions}

Despite many PHM frameworks that have been developed over the past decades, the success is still limited to detecting and classifying faults. Although some works reported a high accuracy in predicting the RUL, their focus mainly on components that have a low complexity such as battery and suspension. The reason is that, for these components, physicbased models are possible to be composed. The prediction accuracy, therefore, is commonly higher in comparison with the data-driven method. For the other components and the entire vehicle, building a robust PHM platform remains a big challenge. The bottleneck is due to two main reasons: the complexity of the (sub-)system and the available data. While the high system's complexity leads to many difficulties in developing model-based methods, the lack of data causes difficulties in applying data-driven approaches. To overcome this bottleneck, researchers should focus on developing hybrid approaches. Moreover, open data sources should be available to all researchers. For many reasons, data might be accessible within particular companies and users, but it is restricted for many other researchers. Making these data sources available for all researchers will reduce the remaining effort necessary for building a robust PHM platform. In addition, due to rapid development of sensor technology, remote communication and computational capabilities, standardized hardware/software interfaces, and smartphones, it should be possible to develop a robust PHM app on the smartphone or on the car dashboard for critical subsystems or components. This app will be considered as a self-management tool for the users, so they can actively manage the maintenance scheduling for their vehicle by themselves in the most economic manner.

\section{PHM IN THE AEROSPACE INDUSTRY}

\subsection{Introduction}

Due to the high availability expectations from aircraft operators and clients and the high costs incurred for maintenance, when an aircraft is out of service (Vianna, Rodrigues, \& Yoneyama, 2015) or Aircraft On Ground (AOG), as well as the supportability, testability and realibility of modern aircraft (L. Yang, Wang, \& Zhang, 2016), PHM systems play a significant role in the aerospace industry, from which it originated in the first place. Nowadays, it is very challeng- ing for the industry now to keep its costs as low as possible and to generate maximum revenue, since the last decade has been turbulent for the aviation industry owing to the unprecedented rise in its commodities due to inflation (Paul et al., 2008), as well as due to the fluctuation in the price of fuel. Regarding the latter, IATA published that in 2017 the airline industry's estimated fuel bill reached 149 billion USD, more than 3 times the figure of 2003 (estimated at 44 billion USD) (IATA, 2018). The industry has to ensure that its asset utilization is optimum and therefore, the maintenance management system of the existing aircraft needs to be precise in order to ensure that the aircraft spend maximum time in the air so as to make the best use of its machinery. This is due to the fact that maintenance is extremely expensive, mainly due to the price of spare parts. As a result, one wants to maximise the use and exploit the remaining life of the installed parts, keeping them in operation by maintining and repairing them until they exhaust their life limit and need to be replaced. This is the role of PHM; to make sure that this happens and that no part is exchanged prematurely. The notice of pending equipment failure allows for sufficient lead-time so that necessary personnel, equipment and spare parts can be organized and deployed, thus minimizing both equipment downtime and repair costs, and optimizing maintenance. Integration is one of the trends of PHM systems, which means that PHM systems of engine and other aircraft parts are integrated with aircraft PHM system (Shufen \& Wanying, 2013). To the best of our knowledge, however, there is no generic PHM framework and architecture enabling communication and integration with the various contributing systems (R. Li, Verhagen, \& Curran, 2018), as well as no uniform design framework of aviation PHM systems between countries (L. Yang et al., 2016) and even between carriers/operators. In addition, a systematic method has yet to be established for developing and deploying a PHM system, as the current ones are application or equipment specific (Lee et al., 2014).

Among all the frameworks the most mature system is that of the F35 aircraft, which constitutes the double-deck architecture. Using this multilayered framework, the system integrates the airplane airborne information, and sends the necessary information to the ground controls. This integrated health management system determines the safety of the aircraft and allows for the state management and maintenance guarantee (S. Li, Zhang, \& Wang, 2017). Another predictive maintenance system, for a wide range of helicopters flown by the military (rotocrafts), is called HUMS (Health and Usage Monitoring Systems), developed by UTC Aerospace Systems. This system can detect several different types of issues using vibration analysis, ranging from shaft unbalance to gear and bearing deterioration. In civil aviation the typical representatives are the Airplane Health Management (AHM) system of Boeing (L. Yang et al., 2016), the AIRcraft Maintenance Analysis (AIRMAN) system of Airbus and a more 
recent addition, namely, aircraft real-time health monitoring system (AiRTHM) (L. Yang et al., 2016). For more detailed information on these specific systems we refer the interested reader to (Company, 2006) (Boeing) and (Holzer, 2011), (Drappier, 2008), (Itier, 2007) (Airbus).

There is also a lack of standards for PHM system development, data collection and analysis methods and data management, although the PHM4SMS (Prognostics and Health Management for Smart Manufacturing Systems) of NIST (National Institute of Standards and Technology) serves in designing such standards (Vogl, Weiss, \& Donmez, 2014). Particularly in the aircraft industry, the published standards for the guidance for PHM systems development is MSG-3, developed by the Maintenance Steering Group (MSG) of the Air Transport Association (ATA) and is titled "Operator / Manufacturer Scheduled Maintenance Development". It is used for developing maintenance plans for aircraft, engines, and systems (Air Transport Association of America, 2013) before the aircraft is in service and it also helps in improving safety while at the same time reducing unnecessary maintenance tasks (Vogl et al., 2014).

This chapter is intended to familiarize the reader with the PHM systems in the aerospace industry, by introducing concepts, presenting examples and discussing research opportunities.

\subsection{Classification of Sensors of the Gas Turbofan Engine}

Here we briefly classify the most common and informative measurements of a turbofan engine. An exhaustive list of sensor measurements of the entire airframe and of the stations of a turbofan engine is out of the scope of this review paper. The authors decided to emphasize on the turbofan engine alone, due to the fact that it is the core of the aircraft and one of the most, if not the most, expensive asset of the airframe. Furthermore, this is a starting point for researchers in the quest for informative measurements. In the rest, we classify them by type and by function.

In Table 4 we provide a classification of the most common turbofan sensors based on their type and in Table 5 we present a classification based on their application. We should note here that $\mathrm{N} 3$ below, is not applicable in all engines.

\subsection{PHM Methods in the Aerospace Industry}

In this section we will give an overview of various PHM methods used in the aerospace industry. To be more specific, as stated in the introduction, CBM systems are founded upon the ability to infer equipment condition using data collected from sensors on monitored systems. In aerospace, these systems could be engines, thrust reversers, avionics, flight controls, fly-by-wire, landing gear, braking, Environmental Control Systems (ECS), electrical systems and auxiliary power
Table 4. Turbofan sensors classified by type

\begin{tabular}{|l||l|}
\hline Types & Sensors \\
\hline \hline \multirow{5}{*}{ Temperature } & Oil temperature, Total air/gas temperature \\
& Static air/gas temperature, \\
& Nacelle temperature, \\
& Exhaust gas temperature (EGT) \\
\hline \multirow{3}{*}{ Vibration } & Core vibration, Fan vibration, \\
& Core phase angle, \\
& Fan phase angle \\
\hline \multirow{3}{*}{ Pressure } & Total air/gas pressure, \\
& Static air/gas pressure, \\
& Oil pressure \\
\hline Spoll Speed & Core speed (N2), Fan speed (N1), N3 \\
\hline & Fuel flow, Oil quantity, Altitude, \\
& Mach number, \\
& Variable bleed valve (VBV) position, \\
& Nacelle Anti-ice, \\
& Wing Anti-ice, \\
& Variable stator blades \\
& (VSV) position \\
\hline
\end{tabular}

Table 5. Turbofan sensors classified by application

\begin{tabular}{|l||l|}
\hline Functions & Sensors \\
\hline \hline Gas Path & $\begin{array}{l}\text { Total air/gas pressure, Static air/gas } \\
\text { pressure, Total air/gas temperature, } \\
\text { Static air/gas temperature }\end{array}$ \\
\hline Engine Oil & $\begin{array}{l}\text { Oil temperature, Oil pressure, Oil } \\
\text { quantity }\end{array}$ \\
\hline Engine Balance & $\begin{array}{l}\text { Core vibration, Fan vibration, Core } \\
\text { phase angle, Fan phase angle }\end{array}$ \\
\hline Stalling/Surging & $\begin{array}{l}\text { VBV position, Wing anti-ice, Nacelle } \\
\text { anti-ice, VSV position }\end{array}$ \\
\hline Thrust Setting & $\begin{array}{l}\text { Engine pressure ratio (EPR), Fan } \\
\text { speed (N1), Core speed (N2), N3, Fuel } \\
\text { flow }\end{array}$ \\
\hline Exhaust & Exhaust gas temperature (EGT) \\
\hline Flight Envelope & Altitude, Mach number \\
\hline
\end{tabular}

units, to name a few. For each system there are also numerous sensors, which reflect their components' state and the overall system health. For example, the current Airbus A350 model has a total of around 6,000 sensors across the entire plane and this number will increase as big-data analytics software and broadband links become more affordable (Shih \& Yang, 2014).

In the following sections we discuss prognostic and diagnostic methods used in aviation as they are crucial for safety, customer satisfaction and the airline revenue. We will emphasize more on prognostic applications in the industry, as this type of predictive analytics is common across all fields of industry, but is particularly valuable in commercial aviation. In addition, as mentioned previously, diagnostics is included in prognostics and thus, we can consider prognostics as a natural extension of diagnostics. After all, ones needs the latter to find the former (Sikorska, Hodkiewicz, \& Ma, 2011b). Thus, we can consider the term prognostics to have a broader definition and enclose activities such as supervise, 
monitor, detect and determine initial degradations, as well as make fault/failure predictions.

Before we proceed it is important to disambiguate two terms, which we will be seeing a lot below and are (oftenly) a source of confusion. These are fault and failure. The former implies that the system under observation is still operational, but cannot continue operating without any maintenance action, otherwise it will cease operating, resulting in a failure.

\subsection{Applications of Prognostics Model-based Methods in the Aerospace Industry.}

Celaya et. al, (Celaya, Saha, \& Wysocki, 2009) demonstrated the feasibility of detecting failure precursors in semiconductor device behavior and using particle filtering (PF) which is an intelligent prediction framework to derive RUL estimates. Specifically they apply their method on the isolated gate bipolar Transistor (IGBT), which forms the backbone of avionic systems and plays a crucial role in vehicle controls, communications, navigation and radar systems. They underline the importance of the estimation of an RUL probability density function (PDF), rather than a single value given by the meantime-between-failure (MTBF) approach. In (Daroogheh, Meskin, \& Khorasani, 2014), Daroogheh et al., extended the particle filtering scheme in order to predict the future behavior of nonlinear dynamical system states and parameters based on the observation forecasting concept with time series methods. Specifically, they developed a fixed-lag dynamic linear model with an adaptive length moving window for time series forecasting using a time-varying autoregression-moving-average (ARMA) model with fixed and variable model orders. The proposed method is applied for prognosis of gas turbine engine health parameters for the next 60 steps ahead. In the same context, (Bolander, Qiu, Eklund, Hindle, \& Rosenfeld, 2009), Bolander et. al presented a model-based RUL prediction for aircraft engine bearing prognosis, in which models are updated by utilizing diagnostic data as a source of additional knowledge in order to reduce the uncertainty in the RUL prediction. The RUL prediction method is based on a particle filter approach with Bayesian updating. Modelbased prognostics and diagnostics are effective even in the absence of component characteristics. In this view, in a recent paper by Zhang et al. (M. Y. Zhang, Liu, Hanachi, Yu, \& Yang, 2018), the authors showed the ability and effectiveness of even a generic-developed model-based approach for the degradation of the auxiliary power unit (APU), in the absence of component characteristics. Below we will review this work in more detail.

In (M. Y. Zhang et al., 2018), Zhang et al. monitor the starter degradation of APU, by designing, among others, a physicsbased model. An APU, is a small gas turbine which provides pressurized air to start the main engines and electric power for the aircraft before the main engines start. To initiate the
APU, an electric motor called the starter is used to provide the power required for running the gas turbine. If the starter is degraded, its mechanical power to accelerate the gas turbine decreases and eventually the APU fails to start. This can cause serious consequences such as AOG (Aircraft On Ground), thus delays and potential safety hazards, since if there is a loss of thrust in flight, the APU can be used to start the main engines again.

Since the starter is connected to the gas turbine, it will affect the performance of the APU if it gets degraded. Thus, the APU gas-path measurements collected from the aircraft's sensors can be used to assess the state of the starter degradation. In this paper the authors select the Exhaust Gas Temperature (EGT) as the relevant measurement for the starter degradation, as it is measured by the majority of the control systems (M. Y. Zhang et al., 2018). In this view, they constructed a physics-based model that estimates the EGT of the APU based on thermodynamic principles, to detect anomalies. To do this, they also modeled the two main anatomical components of the APU, the compressor and the turbine. Due to the lack of parameters and component characteristics, they adopted a generic method to construct the models, which we will present below.

For the development of the compressor model the authors used the Buckingham $\pi$ theorem. They show that they can determine the corrected mass in flow through a known relationship between the mass inflow of the compressor and the compressor inlet temperature, pressure, shaft speed, flow coefficient and an empirically determined flow coefficient factor. In addition to showing how to calculate the compressure pressure ratio, based on compressor work. Furthermore it is shown how the polytropic and isentropic efficiencies can be calculated. As a result they could determine the corrected mass flow, pressure ratio and the isentropic (or adiavatic) efficiency. The latter is a parameter to measure the degree of degradation of energy in steady-flow devices. Regarding the turbine model, based on field expert knowledge that the mass flow of the turbine relies mostly on the pressure ratio of the turbine and little on the shaft speed, they could approximate it as a function of the turbine pressure ratio. Similarly, the turbine pressure ratio can be calculated based on the compressor pressure ratio. Thus, with this, they could determine the corrected mass flow, as well as the pressure ratio of the turbine.

With the aforementioned measurements in hand along with the flow compatibility and energy conservation principle, the gas turbine transient model is finalized. Flow compatibility assumes that mass flow through the turbine is equal to that through the compressor. The EGT can be calculated thus through the model, given as inputs the shaft speed at peak EGT point $N_{\text {peak }}$, ambient temperature $T_{01}$, and the altitude that can be used to estimate the ambient pressure $P_{01}$. 
This results in the following overall dependency:

$$
E G T_{\text {cal }}=E G T_{\text {cal }}\left(T_{01}, P_{01}, N, \alpha, P W_{s}\right)
$$

where $\alpha$ is the angular acceleration and $P W_{s}$ is the starter output power.

For this study, data were collected from an Airbus A310 fleet, including 14 starter nominal cases and 12 starter degraded cases. During each starting cycle of the APUs, the authors recorded four parameters. Namely, peak Exhaust Gas Temperature $E G T_{\text {peak }}$ and the aforementioned $N_{\text {peak }}, T_{01}$ and the altitude. Based on these measured data and Eq. (1), the model is constructed recursively as in Fig. 5.

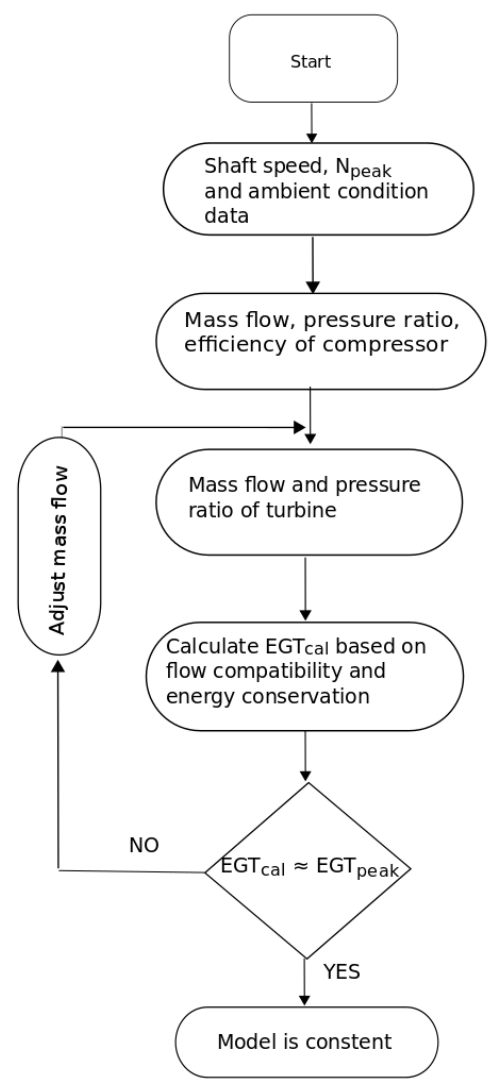

Figure 5. Procedures for constructing the physics-based transient model. Adapted from (M. Y. Zhang et al., 2018).

The physics-based model was implemented to both nominal cases and degraded cases. In figure 6 of the original paper, we observe the EGT estimation based on the approach the authors took, for the nominal condition.

There it can be seen that the physics-based model can effectively estimate the EGT in a nominal case starter and although there are differences between the measured data and the esti- mated EGT values, the model has clearly predicted the variation trend of the EGT. At this point we should also take into account that even though the authors dealt with a lack of component characteristics, the results are acceptably accurate, proving the effectiveness of model-based methods, even in such a generic design.

Subsequently, the authors implemented the model in the degraded - starter cases. In figure 8 (a) and (b), of the original paper, we can see the EGT estimations of the model and the actual measured data in cases I and II. The models estimate the EGT assuming the starter is in nominal condition, while the measured data are collected with a degraded starter. In both figures there are distinctive patterns, which indicate the large deviations between the estimated values and the measured values. This can be seen for case 1 after and around cycle 140 and for case 2 after and around cycle 370 . The reason behind this pattern is, as stated in (M. Y. Zhang et al., 2018), more fuel is pumped in the APU to compensate for the diminished turbine acceleration, due to the degraded starter. This increased fuel flow results in higher EGT values, compared to the nominal condition, under the same shaft speed. This increasing deviation is an indicator of the incremental deterioration of the starter and gives us an idea of what we mentioned earlier. Namely, residuals are large in the presence of actual problems and malfunctions and small when there are normal disturbances, noise and perhaps modelling errors.

The previous example serves to justify in a comprehensive manner the power of a model-based approach in PHM. In many situations however, the complexity of the systems under observation makes it impossible to grasp, even in a generic way, this complexity and design robust and accurate models which can be used for prognostic purposes (Dragomir, Gouriveau, Dragomir, Minca, \& Zerhouni, 2009). Nonetheless, it is often the case that there exists historical data, which capture the behaviour of measured signals or extracted features from the incipient fault stage to even equipment failure. In these cases, data-driven methods should and can be utilized. In the next section we will discuss these approaches.

\subsection{Applications of Prognostics Data-driven Methods in the Aerospace Industry}

Recently in (M. Y. Zhang et al., 2018), Zhang et. al, designed a back-propagation, feedforward neural network to assess the starter degradation of the APU using its gas-path measurements. Feedforward NNs are the simplest form of artificial neural networks where information moves in only one direction from input nodes to output nodes. In a recent paper by Ma et al. (Ma, Lu, Zerhouni, \& Cheng, 2018) the authors proposed an effective deep learning method, termed stacked denoising autoencoder (SDA), for health state classification of aircraft engines considering the environmental 
noise. SDA proved to be effective in terms of cognitive computing and pattern classification theory. Furthermore, the proposed method beats its rivals, in terms of feature extraction due to the benefits of its deep architecture with a data destruction process that is effective for robust feature representation, where high-order features and shared representations can be learnt from the input samples by unsupervised self-learning. The feasibility of the proposed method was demonstrated using the 2008 PHM challenge datasets (see (Ramasso \& Saxena, 2014)). Continuing in this domain, Zhong et. al (Zhong, Li, Lin, \& Zhang, 2018) designed a gated recurrent neural network (GRU Network) to predict the EGT of a turbofan aero-engine. This method could address the time series and nonlinear characteristics simultaneously by the GRU blocks. The proposed algorithm was compared to five other single prognostic methods, namely, an artificial NN (ANN), support vector regression (SVR), extreme learning machine (ELM) and ensemble prognostic methods random forests-based ELM (RF-ELM) and average aggregation ELM (Avg-ELM). The proposed method achieved the best prediction accuracy and acceptable prediction stability. Recurrent NNs, developed in the $80 \mathrm{~s}$, are a class of NNs that capture time dynamics. They constitute a superset of the traditional feedforward NNs that can process information across time steps. Specifically, due to their internal state (memory) they can process a sequence of inputs, granting them the ability to model temporal dependencies and are thus suited for tasks in which input and/or output consist of sequences of points that are not independent. For a more thorough understanding of RNNs we urge the interested reader to (Lipton, 2015). Also in (Vatani, Khorasani, \& Meskin, 2015), Vatani et. al predicted the degradation trends of a gas turbine engine by studying their effects on sensored data (i.e. temperature) by using an RNN as a first approach, as well as a nonlinear autoregressive model with exogenous input (NARX) neural network architecture. In (Zou, Ma, Fang, \& Yi, 2011), Ke$\mathrm{Xu}$ et al. designed a particle-swarm optimized NN for spacecraft prognostics. In (X. Li, Ding, \& Sun, 2018), Li et. al use a deep convolution NN (DCNN) for estimating the RUL and they demonstrate the effectiveness of their method using the C-MAPSS dataset for aero-engine unit prognostics. Convolutional neural networks (CNN) are a class of deep, feedforward NNs mostly used in analysis of visual imagery, that exploit the local dependencies of visual information (Lipton, 2015). For a more thorough understanding of CNNs and their mathematical formulation, we direct the reader to ( $\mathrm{Wu}$, 2017).

Neural networks allow the investigation of complex systems without the need for any knowledge or assumption about system structure. They are sophisticated modelling techniques capable of modelling problems that are analytically and inherently difficult and for which conventional approaches are not practical, including complex physical processes with non- linear, high-order, and time-varying dynamics (Ahmadzadeh \& Lundberg, 2014). NNs however (at least standalone) do not take into account uncertainty bounds arising from different sources like process noise, measurement noise and an inaccurate process model.

In contrast to NNs, relevance vector machines (RVM) and Gaussian process regression (GPR) take into account the width of the uncertainty bounds in addition to providing damage trajectories (Goebel, Saha, Saxena, \& Field, 2008). RVM (Tipping, 1999) is a Bayesian formalism representing a generalized linear model of identical functional form of the support vector machine (SVM). Although SVM (Vapnik, 1995) is a state-of-the-art technique for classification and regression, RVM is able to generate probabilistic outputs in a Bayesian framework that make more sense in RUL estimation applications and futhermore uses a lot kernel functions for comparable generalization performance (Goebel, Saha, Saxena, $\&$ Field, 2008). A GP is a collection of random variables, any finite number of which have a joint Gaussian distribution. The distribution of a Gaussian process is the joint distribution of all those (infinitely many) random variables, and as such, it is a distribution over functions with a continuous domain, e.g. time or space. In (Goebel, Saha, Saxena, \& Field, 2008) the authors evaluate NN-based approach, RVM and GPR for their prognostic capabilities on a test stand involving rotating equipment in an aerospace setting. In the paper, however, there is no clear winner, since each of the algorithms came up with its own current state estimates which were not close to each other. The conclusion states that even though these algorithms can learn the dynamics of the process from sparse and noisy data fairly well, the RUL estimates depend significantly on the current state estimation.

Other approaches used are methods from time-series analysis. The autoregressive moving average (ARMA) model forms a class of general linear models used in modelling and forecasting of time-series. It is comprised by two parts, namely one for the autoregression (AR) and the second for the moving average (MA). It is a powerful forecasting methodology that is able to capture trends found in a time series and projects its future values. In a recent paper by Baptista et. al (Baptista et al., 2018), the authors integrate the ARMA methodology with data-driven techniques, to predict fault events on a real industrial case of unscheduled removals of the engine bleed valve (EBV), based only on life-usage data (maintenance event data). EBV is used in most designs as a regulator for the flow that goes to the ECS and the anti-icing systems of the aircraft. The authors proposed a method in which they feed the entire past fault event history into the ARMA model and the output is then used as a feature that integrates with the datadriven model. The data-driven modeling gives further insight into the forecasting outcome from ARMA and improve its accuracy and efficiency. From the data-driven methods they used, in addition to ARMA (NN, k-nearest neighbors (KNN), 
random forest (RF), support vector regression (SVR), generalized linear regression (GLM)) the SVM produced the best overall results. In a similar manner, $\mathrm{Su}$ et. al used in $(\mathrm{Su}$, Zhang, \& Zhao, 2014) least squares support vector regression with sliding ARMA forecasting to model the non-linear time series. They demonstrate their method on a practical case study for the US-made F-16 fighter.

However, ARMA models are applied in cases were data show evidence of a stationary stochastic process. This means that the time series' statistical properties are all constant over time. A stationary series has no trend. That is, its variations around its mean have a constant amplitude, and it wiggles in a consistent fashion, i.e., its autocorrelations remain constant over time. Equivalently, short-term random time patterns always look the same in a statistical sense. If the contrary stands, its generalization, the Autoregressive Integrated Moving Average (ARIMA) model can be adopted. The "Integrated" indicates that the data values have been replaced with the difference between their values and the previous values, to transform the time series to a stationary one. In a recent paper by Ordóñez et al. (Ordez, Snchez Lasheras, Roca-Pardias, \& Juez, 2019), the authors combine time series analysis methods (ARIMA) to forecast the values of the predictor variables with machine learning techniques to predict RUL of aircraft engine for more than one period ahead, from those variables.

Another important category of data-driven models used are graphical models, which denote the conditional independence structure between random variables (Chen Xiongzi et al., 2011). In a recent paper, (Banghart, Bian, Strawderman, \& Babski-Reeves, 2017), Banghart et al. utilize Bayesian networks (BN) to estimate the risk of the landing gear system, cockpit warning/caution annunciator panel and the environmental control system turbine assembly of the Northrop Grumman EA-6B Prowler military aircraft. BN is a probabilistic graphical model that represents a set of variables and their conditional dependencies via a directed acyclic graph (DAG). Nodes represent variables, while arcs represent probabilistic relationships. For example, engine blade damage impacts non mission-capable time, thus an edge/arc is drawn from the respective nodes. It is a combination of graph theory and probability theory. It is a representation of a joint probability distribution defined on a finite set of random variables that can be discrete or continuous. From a knowledge modeling standpoint, Bayesian networks can be seen as a special knowledge representation system. The advantage of BN lies in the fact that it does not rely on explicit understanding of causal connections within the system(s) under observation, nor identification of sequences of events leading to failure. Furthermore, given their probabilistic nature, BNs prove to be a suitable technique to address the inherent uncertainty of RUL estimation. In the same view, Ferreiro et al. in (Ferreiro \& Arnaiz, 2010) use BN as a predicting technique and demonstrate their effectiveness by representing a physical-model for aircraft brake wear, originally developed by British Aerospace Systems. They fit it to the available data (aircraft weight, landing velocity, brake operation during landing, flap position and initial brake temperature) about flight conditions extracted from the operational plan of the aircraft. Although in this example the causal connections are based on understanding a physical-system, the general idea is that $\mathrm{BN}$ can be successfully used in prognosis also, instead of diagnosis. A subclass of $\mathrm{BN}$ are the so-called dynamic bayesian networks (DBN), which relate variables to each other, over adjacent time steps. They can be considered simply as BN for the modelling of time-series data (Ghahramani, 2001). A specification of DBN are the hidden Markov models (HMM), which have been applied to prognostic problems in aviation.

HMM is a stochastic process model, characterized by a doubly embedded stochastic process with an underlying hidden stochastic process that can be observed through some probabilistic behaviour. The latter justifies the word hidden. It is also a powerful tool for RUL estimation. HMM is furthermore a parametric model with some distinct characteristics: it can not only reflect the randomness of machine behaviour (i.e., sensor measurements) but also reveal hidden states and changing processes (Ahmadzadeh \& Lundberg, 2014). For a more thorough understanding we direct the reader to (Paul A. Gagniuc, 2017). In this view, in (Bechhoefer, Bernhard, He, $\&$ Banerjee, 2006), the authors investigate the use of a Hidden semi-Markov model (HSMM) to predict the RUL of the shaft of utility helicopters until failure. The difference between a HSMM and AMM is that in the latter the amount of time the process spends in a state before making a transition is identical to 1 (for the discrete case) or exponentially distributed (for the continuous case). Thus, for semi-Markov processes, an upcoming transition's distribution is described by a product of an arbitrary PDF for the waiting time and a categorical distribution for the next state. The arbitrary condition for the PDF, removes the memorylessness property of the process and as such, the process is Markovian only at the specified jump instants. Dong et al. in (Dong, He, Banerjee, \& Keller, 2006), proposed a HSMM for fault classification application for UH-60A Blackhawk main transmission planetary carriers and prognosis of a hydraulic pump health monitoring application. They compare HSMM with HMM and conclude that the former are capable of identifying the faults under both test cell and on-aircraft conditions while the performance of the HMM is not comparable with that of the HSMM, while also the HSMM-based methodology can be used to estimate the RUL of equipment. However, HMM have some inherent limitations. One is the assumption that successive system behaviour observations are independent and the other is that the Markov assumption that the probability in a given state at time $t$ only depends on the state at time $t-1$ is clearly untenable in practical applications (Ahmadzadeh \& Lundberg, 
2014).

In the same context, time-series analysis methods, which we referred to before, have been combined with HMM. Specifically, AR models have been combined with HMM, in what is called an autoregressive hidden markov model (ARHMM) (Poritz, 1982), initially proposed for speech recognition. Here the observations are drawn from an autoregression process (linear prediction) (Rabiner, 1990). In this view, Juesas et al. (Juesas, Ramasso, Drujont, \& Placet, 2016) developed a variant of ARHMM, named autoregressive partiallyhidden markov model (ARPHMM) for fault detection and prognostics of equipment based on sensors' data. The authors considered a modification of the learning procedure of the ARHMM, by integrating prior knowledge on latent variables. Their method was demonstrated on an instance of the CMAPSS dataset. They compared their approach, on the aforementioned dataset, against RULCLIPPER (Remaining Useful Life estimation based on impreCise heaLth Indicator modeled by Planar Polygons and similarity-basEd Reasoning) (Ramasso, 2014) (see also Section 8), and SWELM (Summation Wavelet Extreme Learning Machine) (Javed, 2013). The results are promising, in the sense that they are comparable to RULCLIPPER, and, on average, better than SWELM. It is interesting however, as the authors point out, that using an ensemble between ARPHMM and SWELM outperforms RULCLIPPER, showing a direction towards developing ensemble approaches made of complementary and advanced prognostics algorithms.

Finally, a method with which we would like to conclude this section is the use of Neuro-Fuzzy systems (NF) for prognostics. NF systems are neural network-based fuzzy systems, with the latter being a nonlinear mapping of an input data vector with a scalar output. Fuzzy logic is based on Zadeh's fuzzy set theory (Kan, Tan, \& Mathew, 2015). For a better understanding of fuzzy logic and neuro-fuzzy systems, we refer the reader to (Ross, 2010) and (Abraham, 2005), respectively. In (Chen, Vachtsevanos, \& Orchard, 2012), the authors propose an integrated adaptive neuro-fuzzy inference systems (ANFIS) and high-order particle filtering, which forecasts the time evolution of the fault indication and estimates the probability density function of RUL. The ANFIS is used to model the fault propagation trend and the high-order PF integrates the ANFIS, as an $m$-th-order hidden Markov model, to carry out long-term predictions and estimate the RUL PDF via a set of particles with associated weights. They apply their method on vibration data from the main gearbox of a UH60 helicopter subjected to a seeded carrier plate crack fault and show that its prediction accuracy is higher than that of both the conventional ANFIS predictor and the particle-filterbased predictor where the fault growth model is a first-order model that is trained via the ANFIS.

The advantages of data-driven methods for prognostics are that they can be deployed quicker and cheaper compared to other approaches such as model-based. This is true especially when the monitored system is sufficiently complex, such that developing an acceptably accurate model is prohibitively expensive. Another advantage of data-driven methods is their versatility and cost in terms of development, as well as that they refer to a wider audience of researchers than modelbased methods. Furthermore, they can provide a system-wide coverage in comparison to model-based methods, which usually have a more narrow scope in order to model a particular system or component. Finally, they are popular due to their ability to transform high-dimensional noisy data into lower dimensional information for diagnostic/prognostic decisions (Dragomir et al., 2009).

In view of this, we are presenting part of (Vatani et al., 2015), to allow the reader to understand the aforementioned. In (Vatani et al., 2015) the authors predict the degradation trends of a gas turbine engine by studying their effects on sensor data (i.e. temperature) by using an RNN, as one the different approaches. We will describe this approach here and we refer the interested reader to the original paper for all the explicit details and methods.

The authors' goal is to predict the trend of a single-spool turbofan engine, and for this purpose, identify the major reasons for engine degradation. They consider the effects of fouling and erosion, which constitute the most significant long-term effects on engine performance, when compared to the others, and study their effects only for take-off, since this is the flight phase in which the effects and growth of degradation are more prominent. We will cover here the effects of fouling and its effects on the compressor as studied by the authors.

For the fouling degradation modeling they define initially a fouling index (FI), to represent the fouling effects on the engine performance. The FI is modeled as a linear degradation. Secondly, they introduce a degradation index (DI) to quantify its relationhsip to mass flow capacity and efficiency and define the rate of change in mass flow capacity of the compressor and its efficieny, as well as the degradation gain in each cycle of the mass flow capacity and efficiency of the compressor. It should be pointed out that the objective is not to train the RNN to identify the dynamics of the engine, but instead to train the networks to represent, learn and predict the dynamics of the degradation process on the engine variables and how it grows in the system as the flight cycles build up.

For the data generation, they specifically simulate the groundroll phase, which includes taxiing until lift-off, and all simulations are performed under standard operating conditions (ambient temperature and pressure assumed to be at the standard sea-level at take-off). The results related to the degradation modelling and the changes to the engine output parameters due to different degradation levels are compared with the GSP 
10 (Gas turbine Simulation Program - National Aerospace Laboratory NLR) single spool turbojet model. They picked this phase because the engines operate from their idle position to their maximum power and the effects of degradation are more significant. In the simulations every flight cycle takes $3000 \mathrm{~s}$, out of which the take-off is $20 \mathrm{~s}$. The compressor pressure and temperature are recorded, as well as the spool speed and the fuel flow rate. In this work the effects of the turbine temperature (TT) variations is chosen as a suitable indicator, due to the fact that changes in the compressor health parameters (compressor flow rate and compressor efficiency) affect the gas path measurements. Finally, to manage the uncertainty of the prognosis, they define upper and lower bounds. When the upper bound of the prediction variable meets a specified threshold, one can declare that the engine should be taken for maintenance.

For the fouling effects they generate data for $1 \%$ fouling to train the RNN. Based also on the assumption that fouling is a soft degradation and as such, does not change the engine's performance in one flight (drastically), they feed the network with just the 12th second snapshot.

Initially, they consider an RNN with a 4-5-1 architecture i.e., 4 input nodes, 1 hidden layer with 5 nodes and 1 ouput node. They then do a 3 step-ahead prediction. The statistical error results are given as (mean error) $\mu=0.177$ Kelvin, (standard deviation of the prediction error) $\sigma=1.384$ Kelvin, and (root mean squared error) RMSE $=1.343$ Kelvin. The results are shown in figure 3 in the original paper. There, we can see that most of the TT values lie in the prediction bounds as predicted by the RNN and only two points fall out of the bands, giving a $92.6 \%$ of points inside. They further consider a 10 stepsahead prediction with the $1 \%$ fouling using a 6-5-1 architecture, i.e., 6 neurons in the input layer, 5 neurons in the hidden layer, and 1 neuron in the output layer. The statistical results for the 10 steps-ahead prediction are (mean error) $\mu=-0.338$ Kelvin, (standard deviation of the prediction error) $\sigma=1.810$ Kelvin, and (root mean squared error) RMSE $=2.786$ Kelvin, which represent a reasonable error level, taking into account the prediction horizon. The results are shown in figure 4 in the original paper. In the figure one can observe that several actual (measured) data points have exceeded beyond the bounds and only $65 \%$ lie inside it. They conclude that the achieved error level for the second RNN meets the expected specifications as the maximum error is 4 degrees Kelvin which is less than $1 \%$ of the range of $1400 \mathrm{~K}$, implying that the prediction is $99 \%$ accurate. They also conclude that the first RNN is the most suitable network for predicting a $1 \%$ fouling effect on the TT. Finally, with the prediction results one can obtain the system RUL when the gas path measurements reach prespecified thresholds.

To conclude this section, we must also point out some disadvantages of data-driven methods. One important is that they are data-hungry, that is, they require a substantial amount of data for training and thus, they are highly-dependent on the quantity and quality of the system's operational data. There is also commonly a lack of a procedure to obtain the training data and there is further a lack of run-to-failure data, for the methods to learn from. Due to this, applications in the literature just use experimental data for model training and thus these approaches may have wider confidence intervals than others. (Dragomir et al., 2009).

To counter these limitations, including the limitations of model-based techniques, fusion approaches have been designed, to combine their strengths. In the next section, we discuss these.

\subsection{Applications of Prognostics Hybrid Methods in the Aerospace Industry}

Fusion or hybrid-based prognostic methodologies combine the strengths of the model - based and data - driven approaches, in order to estimate the RUL under both operating and non-operating life cycle conditions (Pecht \& Jaai, 2010). By taking advantage of the two respective methods' strengths, hybrid models can achieve robust health prediction results that can lead to a more reliable RUL approximation as compared to only model-based methods. Also, due to the use of a mathematical model, the amount of data required for training purposes are relatively lower than that needed in pure data - driven methods (Daroogheh, Baniamerian, Meskin, \& Khorasani, 2015). A hybrid model thus combines both data-driven methodologies with the knowledge of the system under study.

In a recent paper by Daroogheh et al., (Daroogheh et al., 2015), the authors proposed a hybrid structure for the health monitoring of non-linear systems. Specifically, they fuse particle filtering to estimate the states and health parameters of the system and extend the PF to the future time horizon by utilizing a neural network $(\mathrm{NN})$ as a non-linear forecasting scheme. The authors apply the proposed method to predict the health condition of a gas turbine engine.

Also recently in (Xu, Wang, \& Xu, 2014) the authors proposed a PHM-oriented hybrid prognostics framework for aircraft engines based on the measured sensor data, with a focus on RUL prediction. They are advocates of the idea that for such complicated problems it is difficult to model the complex degradation process, and no single prognostic approach can effectively solve this. Their fusion prognostic method utilizes the aircraft engine sensor data and combines the strengths of both data-driven and experience-based prognostic approaches and eliminates their limitations. In the same view (Xu \& Xu, 2011) design a fusion prognostic model for avionics systems by combining data-driven, model-based and experience-based PHM methods. Their fusion model is 
built upon the optimal linear combination forecast model using absolute value and prediction error as the index of prognostic precision. They conclude that their fusion prognostics can provide RUL estimation more accurate and more robust than either of the algorithms alone (ARMA, SVM, FNN).

In (Byington, Watson, \& Edwards, 2004), the authors designed a prognostics framework for aircraft actuator components. Their methodology includes automated signal processing and neural network tracking techniques, along with automated reasoning, classification, knowledge fusion, and probabilistic failure mode progression algorithms. They implement a fuzzy logic process to quantify the level of damage (damage index) of the system using a predefined set of rules tailored from knowledge of the system and engineering judgment (experience-based method). A physics-based method (Kalman filtering) was then applied to predict the progression of the damage. They demonstrated their method to F/A-I8 stabilator electro-hydraulic servo valves (EHSVs).

In (Heimes, 2008), Heimes, applied an RNN trained by backpropagation method, using extended Kalman filter (EKF) training method to update the weights of the network. The EKF is particularly attractive for RNN training, since it minimizes the number of training iterations and does not require all the training data to be utilized for each training iteration. The RUL for the simulated aircraft engines was calculated by simply averaging the outputs of the three best models. This solution won 2nd place in the IEEE 2008 PHM Conference challenge problem (see (Ramasso \& Saxena, 2014)), in which the contestors where asked to estimate the RUL (remaining operational cycles) of multiple multivariate time-series of engine data.

In (Howard, Mesick, Reuter, \& Roemer, 2001), the authors present an architecture that combines the model-based and data-driven approaches for fault detection, diagnosis, and prognostics for aircraft. It includes a prognostic reasoner that takes as input the outputs from a variety of specialized prognostic algorithms for different systems within the aircraft, and then prioritizes the most probable failure modes. The system uses an integrated model to predict how a probable failure will affect systems throughout the aircraft. It also compares the outputs of the anomaly detector, the diagnostic system, and the prognostic system to see whether the three types of algorithms endorse each other. The authors also include a brief description of some fault detection and diagnostic algorithms that they have used with the architecture, but there is no description of prognostic algorithms.

In (Lasheras, Nieto, de Cos Juez, Bayn, \& Surez, 2015) the authors use a hybrid model, with the meaning that it is usually employed in the field of machine learning and pattern recognition, for predicting the RUL of aircraft engines. They combine the multivariate adaptive regression splines (MARS) technique with the principal component analysis (PCA), den- drograms and classification and regression trees (CARTs). The proposed model does not require information about the previous status of the variables of the engine and it only requires information regarding the current situation of the studied variables and is trained by elements extracted from the engine's sensors representing different levels of health for aircraft engines. They conclude that their model performs better than the classical predictive models (linear multivariate regression model and neural network model with back propagation) applied in recent years for the modeling of RUL.

We decided to present the work of (Daroogheh et al., 2015) to give the reader an example of how a fusion method can work. In this paper the authors apply a hybrid method to estimate the RUL of a compressor of a single spool jet engine, which is being degraded due to fouling phenomena. The single spool jet engine model they used was developed in (Naderi, Meskin, \& Khorasani, 2011), (Naderi, Meskin, \& Khorasani, 2012). Specifically they combine state/parameter estimation using particle filters (PF) (model-based method) with neural networks for observation prediction (data-driven method).

For the model-based part, they implement two parallel filters for the state and parameter estimation tasks. At each time step for the state (parameter) estimator filter, the corresponding parameters (states) are treated as being a known input to the filter from the parameter (state) estimator. Regarding the data-driven part, for the NN, they used a multilayer perceptron (MLP) and RNN and wavelet neural network (WNN). With the use of the NN, they forecasted observations/measurements (data-driven prediction) allowing for the $\mathrm{PF}$ algorithm to be extended to future steps by utilizing the same weight update rule used in the estimation step. For this task, they use the mean squared error (MSE) when new observations are available, in order to validate and if, needed, retrain the network if the MSE exceeded a predefined threshold.

For their application they use as system state parameters, the compressor and turbine efficiency and the compressor and turbine mass flow capacities. They apply a linear degradation model on the compressor health parameters during 1000 cycles of operation causing a drop of $3 \%$ to compressor efficiency and $1.5 \%$ to its mass flow capacity and they define as the critical values 0.7832 for the compressor efficiency and $20.0127 \mathrm{~kg} / \mathrm{s}$ for the compressor mass-flow capacity. The authors use as a ground truth failure cycle (based on the aforementioned critical values) to be at 230 and predict 40 steps ahead from cycle 200 . The latter is because they assume that the required data to train the networks are 200. The results can be seen in the original paper in figure 3 and figure 4.

The presented results indicate that the PF method without integration (thus a standalone implementation) with an observation forecasting module (such as the implemented $\mathrm{NN}$ ), is not able to track the changes in the compressor efficiency cor- 
rectly and has overestimated this health parameter. It is apparent that it cannot be located inside the $+/-99 \%$ confidence interval around the real value. Although for mass flow capacity (figure 3 bottom of the original paper) of the compressor, the PF method has estimated the value of this health parameter inside the confidence bound for most of the times, the direction of the changes in predicted mass flow capacity in the fouling scenario is not correct. However, it is evident that all of the three neural networks are able to predict the compressor health parameters and lie within the $+/-99 \%$ confidence interval around the actual values. In figure 4 of the original paper the distribution of the health parameters is presented, along with the cycle at which the maximum failure probability occurs. They propose that the time at which the first parameter associated with the specific degradation damage reaches its critical value, is the failure cycle. In this example clearly the compressor mass flow capacity is the parameter that first exceeds its critical value. The predicted failure value is cycle 215 (RUL=15 cycles) for the RNN, 220 (RUL=10 cycles) for the WNN and 222 (RUL=8 cycles) for the MLP.

We hope that this paper made it clear to the reader how different methods can be combined and that there is a benefit when using fusion methods in prognostics, especially for non-linear dynamical systems, such as gas turbine engines.

With the hybrid methods, we conclude the review on PHM methodology in the aerospace industry. In the next section we will conclude with this chapter of this literature review.

\subsection{Discussions and Conclusions}

With the number of passengers being carried through air transport (both domestic and international), estimated at 3.979 billion in 2017 (International Civil Aviation Organization \& staff estimates., n.d.), safety and economy for aircraft manufacturers and operators are of greatest significance. Prognostics and Health Management (PHM) allows for this, by performing diagnostics and prognostics on the engines, airframe and other components of an aircraft. The identification and isolation of a fault as well as the predictive diagnostics give the OEMs and airlines the ability to identify and recover from failures and provide them with a heads-up on what to expect. The latter is the essence of PHM and what has directed the transition from traditional health monitoring to health management. Since it foresees a fault or failure, it allows for maximal availability and reliability, shifting away from the time-scheduled maintenance to a condition-based maintenance, thus increasing safety and reducing unnecessary downtime. These early warnings grant the airline also the ability to make a timely maintenance strategy schedule and follow all the necessary procedures for the logistics, such as ordering parts and making agreements with external vendors.

Being around for only a few decades, especially in the aerospace industry, prognostics has still plenty of research potential in this field. It is advisable for future investigations that a design of a standard procedure for obtaining data for PHM is developed. Whether a researcher or an airline is using a model-based a data-driven or a fusion approach, measurement data are needed and are crucial for most methods. It is the view of the authors, that data science should play an indispensable role in such industries. In addition, there is a lack of run-to-failure data for the data-driven algorithms to train on and validate the results. Future research should thus emphasize also on generating data for such purposes, taking into account that, especially in this industry theoretical predictions and methods developed must be verified and validated first before deployed in practical applications.

\section{General discussions and Conclusions}

Prognostics and health management (PHM) is a fairly new discipline that provides actionable information, enabling intelligent decision making for improved performance, safety, reliability, and maintainability of engineered systems, by using real time and historical state information of subsystems and components. Aside from that, its importance lies in that these early warnings grant the user the ability to make a timely maintenance strategy schedule and follow all the necessary procedures for the logistics/supply-chain. It differs from diagnostics in that it can be interpreted as the process of health assessment and prediction, which includes detecting incipient failure and predicting remaining useful life (RUL). It, however, naturally embodies diagnostics which identifies and determines the relationship between the failure mechanism and the failure mode.

In this paper, we have reviewed the recent researches and developments that have been done to apply PHM in the automobile and the aerospace industry. We initially approach the matter generally by presenting the discipline and its significance, before moving on to the applications and methods as they are specifically used in these industries, focusing on the remaining useful life (RUL) estimation.

The main approaches adopted by PHM experts fit into four broad categories. Namely, reliability-based, model-based, data-driven and hybrid or fusion methods. In this review we covered the latter three. Model-based methods assume a mathematical model of the monitored system and use residuals as features. Residuals are the outcomes of the comparisons between the measurements of a real system and the outputs of a mathematical model of that system. Due to the physical understanding of the system, model-based methods are very accurate. However, this close relation to the mathematical model can make them impossible to design, due to the high complexity. Data-driven methods on the other hand, rely on data routinely recorded from the monitored systems and as such they are operational data. Thus, in such cases 
in which data are available the data-driven methods are preferred because of their ease of use These methods adopt techniques from $\mathrm{AI}$, machine learning and pattern recognition. Their main advantage compared to model-based methods, is the fact that they can be deployed faster and cheaper, since they do not assume anything about the underlying relationships of the system under study and therefore they appeal to a wider audience and techniques. Everything comes with a cost though. These methods might be cheaper to deploy, but rely largely on data, which is most often of questionable quality, is rare or not existent or cannot be disclosed. Also, their accuracy might not be as high as that of a model-based approach. Hybrid approaches combine model-based and data-driven approaches strengths while at the same time minimizing their disadvantages. Hybrid approaches are newly developing and promising. However, to the best of our knowledge, except for battery, hybrid approaches have not been widely used in PHM for the automobile- and aerospace industries. We particularly addressed the importance of performance metrics and uncertainty evaluation in PHM. Uncertainties arise from various sources such as: modeling uncertainties, measurement uncertainties, operating environment uncertainties and others alike. Such information is crucial for any prognostic approach and must be validated before being incorporated in PHM applications.

Despite the fact that PHM has been around for just a few decades, plenty of work has been done in estimating the RUL. However, their practical success seems to be still limited in diagnosis, because there are a number of challenges and practical issues to be addressed. For example, even though researchers developed data-driven methods to counter the increasing complexity of systems and components, there is still no standard procedure to obtain data, in terms of a protocol or system. Data are either not integrated centrally, but scattered around different systems, or cannot be disclosed due to security and privacy issues and competition. To add to that, there is also the possibility that data do not even exist due to cost, as for example run-to-failure data of a turbofan engine. Future research should thus emphasize also in generating data for such purposes or when it is not available, taking into account that in field applications theoretical predictions and methods developed must be verified and validated first before practical applications become possible. Finally, for fielded applications another crucial challenge is the real-time (online) RUL estimation. The issue lies in the fact that the developed methods need intensive computational resources, which is in direct contradiction with hardware conditions of on-board computers.

What is more, setting aside the fact that plenty of fundamental work has been done in the context of PHM, the issue still remains that simplifying assumptions have to be made, since the underlying system is too complex. For example, in modelbased, simplifying assumptions are made about the system, in order to be able to mathematically model it. In data-driven, on the other hand, assumptions are made regarding i.e., the shape of the health degradation curve, and in addition results are not always interpretable. While these approaches have given us remarkable results, and still have a lot to offer, we feel the urge to suggest future work towards an area that, we believe, has been overlooked in prognostics, and can overcome some of the shortcomings mentioned above, for the data-driven and model-based approaches. That method is evolutionary computation (EA) and particularly symbolic regression (SR). SR is a subclass of genetic programming (GP), which in turn is a subclass of genetic algorithms (GA), that belong to evolutionary algorithms (EA). SR is a regression technique that searches the space of algebraic expressions, to find the model that best fits the given data, without making any assumptions about the structure of the model (i.e., linear or non-linear). It, instead, evolves mathematical formulas, in the evolutionary computation sense. Such an approach can help mitigate some of the issues mentioned above. For example it can be very effective in uncovering the physics that govern a physicalsystem, such as a turbofan engine, and as a result increases system understanding. In such a case sensor measurements provide us with I/O data. A SR could unveil the underlying mathematical relations, which could then be used as a model-based approach in the standard way, as discussed in this review. Furthermore, by uncovering the generating process of the data, it can provide insight on the quality of the data recorded. What is more, it can alleviate the black-box approach of usual data-driven methods, since it provides a mathematical expression as a result, and thus allowing the model to be better interpreted.

In addition, most of developed PHM approaches follow the modern control theory (MCT); meaning the established models (mathematical, physical or machine learning models) are built on the train datasets then they are validated on the test datasets. Normally, system identification algorithms can be employed to train a model from data, which is then used to design the controller (models and hyper-parameters). Designing a controller by first identifying an open-loop model of the process, and then synthesizing a controller based on the resulting model, would require focusing the attention on reproducing the open-loop behavior of the process first, then selecting a model structure, tune the model parameters, compare the open-loop response of the model on validation data, etc (Selvi, Piga, \& Bemporad, 2018). Therefore, the chosen established models should reflect the desired closed-loop performance in a PHM system and be reproducible by the underlying unknown process when in a closed-loop with the synthesized controller. However, building a model in PHM is neither costly- nor time-consuming, it remains difficult to decide a-priori the level of accuracy/complexity the model should have to meet the desired closed-loop performance. Difficulty in modeling, poor robustness, lack of safety, as well 
as a huge gap between theoretical analysis and practical performance due to un-modeled dynamics and parametric uncertainty, are common phenomena when applying control theory methods in practice (Hou \& Jin, 2014).

Therefore, using data-driven model-free (DDMF) methods would be a new trend in PHM. In these methods, the measurement input/output (I/O) data is directly used for control design and closed-loop system analysis, without any model dynamics involved, thus, DDMF is also called as data-driven control method. (Hou \& Jin, 2014). In DDMF, adaptive control for an unknown nonlinear system with time-varying parameters and time-varying structure is uniformly realized, and the existing difficulties in modern control methods, such as the dependence of controller design on system model, unmodeled dynamics, traditional robustness issues, and other related theoretical problems, are avoided within the datadriven control framework. Several examples of the DDMF frameworks are Virtual Reference Feedback Tuning (VRFT) (Campi, Lecchini, \& Savaresi, 2002), Iterative Feedback Tuning (IFT) (Hjalmarsson, 2002), Simultaneous Perturbation Stochastic Approximation (SPSA) (Spall \& Cristion, 1998), Model-Free Iterative Learning Control (MFILC) (Radac, Precup, \& Petriu, 2015), Model-Free Adaptive Control (Hou \& Jin, 2011; Fliess \& Join, 2013).

Both the theoretical results (Radac, Precup, \& Roman, 2018; Selvi et al., 2018; Guo, Hou, Liu, \& Jin, 2019) incessantly developed and improved in the past two decades, and their promising practical applications in chemical industry (dos Santos Coelho, Pessôa, Sumar, \& Coelho, 2010), healthcare (Meneghetti, Terzi, Del Favero, Susto, \& Cobelli, 2018), machinery (Tan, Lee, Huang, \& Leu, 2001; Radac \& Precup, 2017; Duan, Hou, Yu, Jin, \& Lu, 2019; Stainier, Leygue, \& Ortiz, 2019), and so on, would suggest DDMF to be novel methods for developing systematic and rigorous PHM frameworks. As an outstanding example, in 2014, Ramasso established a RULCLIPPER algorithm for estimating the RUL of physical systems, by utilising health index/health indicator (HI), which reflect the system's health state. In this DDMF framework, the HI are assumed imprecise (IHI) (Ramasso, 2014). RULCLIPPER is comprised of ideas inspired from both computer vision and computational geometry, with IHI data interpreted as vertices of a simple (non self-intersecting) planar polygon. The algorithm relies further on the adaptation of case-based reasoning (see (T. Wang, Jianbo Yu, Siegel, \& Lee, 2008)) to manage the imprecise training and testing instances. The method was validated with C-MAPSS datasets and illustrated that computational geometry seems promising for PHM in presence of noisy HIs. While some similaritybased matching algorithms (case-based reasoning) may suffer from computational complexity, in particular to sort training instances, the proposed algorithm is shown to be efficient with few parameter tuning on all datasets. Additionally, the authors claim that RULCLIPPER is a general algorithm, in- variant to the peculiarities of the datasets that it can be used on.

To conclude, future directions for research in PHM should include the use of data science tools for generating, and effectively disseminating data. This includes also the integration of data sources with smart technologies for the end-users. What is more, there is a need for research in uncertainty representation for online evaluation of prognostics to deal with uncertainties associated with future operating conditions, in order for prognostics to be deployed in the real world.

We surely hope that this review will help researchers in the field to get an overview on recent developments of PHM in the aerospace and automobile industry, as well as a more general picture of PHM and its importance. We believe that PHM related research will continue to grow in the following years, as there is still much to learn on the potential of data, as well as to mitigate all the current shortcomings.

\section{ACKNOWLEDGEMENTS}

This work is part of the research programme Smart Industry SI2016 with project name CIMPLO and project number 15465 , which is partly financed by the Netherlands Organisation for Scientific Research (NWO).

\section{REFERENCES}

Abraham, A. (2005, July). Adaptation of Fuzzy Inference System Using Neural Learning. In J. Kacprzyk, N. Nedjah, \& L. d. Macedo Mourelle (Eds.), Fuzzy Systems Engineering (Vol. 181, pp. 53-83). Berlin, Heidelberg: Springer Berlin Heidelberg. doi: 10.1007/11339366_3

Accident: Southwest B737 near Philadelphia on Apr 17th 2018, uncontained engine failure takes out passenger window. (n.d.).

Ahmadzadeh, F., \& Lundberg, J. (2014, December). Remaining useful life estimation: review. International Journal of System Assurance Engineering and Management, 5(4), 461-474. doi: 10.1007/s13198-0130195-0

Alia, J. B., Chebel-Morello, B., Saidi, L., Malinowski, S., \& Fnaiech, F. (2015). Accurate bearing remaining useful life prediction based on weibull distribution and artificial neural network. Mech Syst Signal Process, 150172 .

Arulampalam, M. S., Maskell, S., Gordon, N., \& Clapp, T. (2002). A Tutorial on Particle Filters for Online Nonlinear/Non-Gaussian Bayesian Tracking. IEEE Transactions on Signal Processing, 50(2), 15.

Ashok, B., Denis Ashok, S., \& Ramesh Kumar, C. (2016). 
A review on control system architecture of a SI engine management system. Annual Reviews in Control, 41, 94-118. doi: 10.1016/j.arcontrol.2016.04.005

Ashok, B., Denis Ashok, S., \& Ramesh Kumar, C. (2017). Trends and future perspectives of electronic throttle control system in a spark ignition engine. Annual Reviews in Control, 44, 97-115. doi: 10.1016/j.arcontrol.2017.05.002

Atamuradov, V., Medjaher, K., Dersin, P., Lamoureux, B., $\&$ Zerhouni, N. (2017). Prognostics and health management for maintenance practitioners - Review, implementation and tools evaluation., 32 .

Banghart, M., Bian, L., Strawderman, L., \& BabskiReeves, K. (2017, July). Risk assessment on the EA-6b aircraft utilizing Bayesian networks. Quality Engineering, 29(3), 499-511. doi: 10.1080/08982112.2017.1319957

Baptista, M., Sankararaman, S., de Medeiros, I. P., Nascimento, C., Prendinger, H., \& Henriques, E. M. (2018, January). Forecasting fault events for predictive maintenance using data-driven techniques and ARMA modeling. Computers \& Industrial Engineering, 115, 4153. doi: 10.1016/j.cie.2017.10.033

Baraldi, P., Compare, M., Sauco, S., \& Zio, E. (2013). Ensemble neural network-based particle filtering for prognostics. Mechanical Systems and Signal Processing, 41(1), 288 - 300. doi: https://doi.org/10.1016/j.ymssp.2013.07.010

Beatrice, C., Guido, C., Napolitano, P., Iorio, S. D., \& Giacomo, N. D. (2011, May). Assessment of biodiesel blending detection capability of the onboard diagnostic of the last generation automotive diesel engines. Fuel, 90(5), 2039-2044. doi: 10.1016/j.fuel.2011.01.013

Bechhoefer, E., Bernhard, A., He, D., \& Banerjee, P. (2006). Use of Hidden Semi-Markov Models in the Prognostics of Shaft Failure. , 7.

Berecibar, M., Gandiaga, I., Villarreal, I., Omar, N., Van Mierlo, J., \& Van den Bossche, P. (2016, April). Critical review of state of health estimation methods of Li-ion batteries for real applications. Renewable and Sustainable Energy Reviews, 56, 572-587. doi: 10.1016/j.rser.2015.11.042

Bolander, N., Qiu, H., Eklund, N., Hindle, E., \& Rosenfeld, T. (2009). Physics-based Remaining Useful Life Prediction for Aircraft Engine Bearing Prognosis., 12.

Brier G.W., \& Allen R.A. (1951). Verification of Weather Forecasts. Boston, MA: In: Malone T.F. (eds) Compendium of Meteorology. American Meteorological Society.

Byington, C., Watson, M., \& Edwards, D. (2004). Datadriven neural network methodology to remaining life predictions for aircraft actuator components. In 2004 IEEE Aerospace Conference Proceedings (IEEE Cat.
No.04th8720) (Vol. 6, pp. 3581-3589). Big Sky, MT, USA: IEEE. doi: 10.1109/AERO.2004.1368175

Campi, M., Lecchini, A., \& Savaresi, S. (2002). Virtual reference feedback tuning: a direct method for the design of feedback controllers. Automatica, 38(8), 1337 - 1346. doi: https://doi.org/10.1016/S0005-1098(02)00032-8

Celaya, J. R., Saha, B., \& Wysocki, P. F. (2009). Prognostics for Electronics Components of Avionics. , 7.

Chen, C., Vachtsevanos, G., \& Orchard, M. E. (2012, April). Machine remaining useful life prediction: An integrated adaptive neuro-fuzzy and highorder particle filtering approach. Mechanical Systems and Signal Processing, 28, 597-607. doi: 10.1016/j.ymssp.2011.10.009

Chen Xiongzi, Yu Jinsong, Tang Diyin, \& Wang Yingxun. (2011, August). Remaining useful life prognostic estimation for aircraft subsystems or components: A review. In IEEE 2011 10th International Conference on Electronic Measurement \& Instruments (pp. 94-98). Chengdu, China: IEEE. doi: 10.1109/ICEMI.2011.6037773

Cheng, S., Azarian, M. H., \& Pecht, M. G. (2010, June). Sensor Systems for Prognostics and Health Management. Sensors, 10(6), 5774-5797. doi: 10.3390/s100605774

Coble, J., Ramuhalli, P., Bond, L. J., Hines, J. W., \& Ipadhyaya, B. (2015). A Review of Prognostics and Health Management Applications in Nuclear Power Plants. , 24.

Company, T. (2006). Benefits of IVHM: An Analytical Approach. In 2006 IEEE Aerospace Conference (pp. 1-9). Big Sky, MT, USA: IEEE. doi: 10.1109/AERO.2006.1656072

Dang, X., Yan, L., Xu, K., Wu, X., Jiang, H., \& Sun, H. (2016, January). Open-Circuit VoltageBased State of Charge Estimation of Lithium-ion Battery Using Dual Neural Network Fusion Battery Model. Electrochimica Acta, 188, 356-366. doi: 10.1016/j.electacta.2015.12.001

Daroogheh, N., Baniamerian, A., Meskin, N., \& Khorasani, K. (2015, June). A hybrid prognosis and health monitoring strategy by integrating particle filters and neural networks for gas turbine engines. In 2015 IEEE Conference on Prognostics and Health Management (PHM) (pp. 1-8). Austin, TX, USA: IEEE. doi: 10.1109/ICPHM.2015.7245020

Daroogheh, N., Meskin, N., \& Khorasani, K. (2013, June). Particle filtering for state and parameter estimation in gas turbine engine fault diagnostics. In 2013 American Control Conference (pp. 4343-4349). Washington, DC: IEEE. doi: 10.1109/ACC.2013.6580508

Daroogheh, N., Meskin, N., \& Khorasani, K. (2014, June). A novel particle filter parameter prediction scheme for failure prognosis. In 2014 American Control Conference (pp. 1735-1742). Portland, OR, USA: IEEE. doi: 


\subsection{9/ACC.2014.6859021}

Dishant, E. S., Er.Parminder Singh. (2017, April). Suspension systems: A review. International Research Journal of Engineering and Technology (IRJET). doi: 10.1007/s00521-017-2986-8

Dong, M., \& He, D. (2007). A segmental hidden semimarkov model (hsmm)-based diagnostics and prognostics framework and methodology. Mechanical Systems and Signal Processing, 21(5), 2248 - 2266. doi: https://doi.org/10.1016/j.ymssp.2006.10.001

Dong, M., He, D., Banerjee, P., \& Keller, J. (2006, October). Equipment health diagnosis and prognosis using hidden semi-Markov models. The International Journal of Advanced Manufacturing Technology, 30(7-8), 738-749. doi: 10.1007/s00170-005-0111-0

dos Santos Coelho, L., Pessôa, M. W., Sumar, R. R., \& Coelho, A. A. R. (2010). Model-free adaptive control design using evolutionary-neural compensator. Expert Systems with Applications, 37(1), 499 - 508. doi: https://doi.org/10.1016/j.eswa.2009.05.042

Downey, A., Lui, Y.-H., Hu, C., Laflamme, S., \& Hu, S. (2019, February). Physics-based prognostics of lithium-ion battery using non-linear least squares with dynamic bounds. Reliability Engineering \& System Safety, 182, 1-12. doi: 10.1016/j.ress.2018.09.018

Dragomir, O. E., Gouriveau, R., Dragomir, F., Minca, E., \& Zerhouni, N. (2009, August). Review of prognostic problem in condition-based maintenance. In 2009 European Control Conference (ECC) (pp. 1587-1592). Budapest: IEEE. doi: 10.23919/ECC.2009.7074633

Drappier, C. J. (2008). A380: Challenges for the Future., 34.

Duan, L., Hou, Z., Yu, X., Jin, S., \& Lu, K. (2019). Data-Driven Model-Free Adaptive Attitude Control Approach for Launch Vehicle With Virtual Reference Feedback Parameters Tuning Method. IEEE Access, 7, 54106-54116. doi: 10.1109/ACCESS.2019.2912902

Ekwaro-Osire, Stephen, Alemayehu, Fisseha M, \& Carlos Gonalves, Aparecido. (2017). Probabilistic Prognostics and Health Management of Energy Systems. Springer International Publishing.

Elattar, H. M., Elminir, H. K., \& Riad, A. M. (2016, June). Prognostics: a literature review. Complex \& Intelligent Systems, 2(2), 125-154. doi: 10.1007/s40747016-0019-3

Ferreiro, S., \& Arnaiz, A. (2010). Prognostics applied to aircraft line maintenance: Brake wear prediction based on Bayesian Networks. IFAC Proceedings Volumes, 43(3), 146-151. doi: 10.3182/20100701-2-PT-4012.00026

Fleming, W. (2001, December). Overview of automotive sensors. IEEE Sensors Journal, 1(4), 296-308. doi: $10.1109 / 7361.983469$

Fliess, M., \& Join, C. (2013). Model-free control. International Journal of Control, 86(12), 2228-2252. doi:
10.1080/00207179.2013.810345

Ghahramani, Z. (2001). AN INTRODUCTION TO HIDDEN MARKOV MODELS AND BAYESIAN NETWORKS. Hidden Markov Models, 34.

Goebel, K., Saha, B., Saxena, A., Celaya, J., \& Christophersen, J. (2008, August). Prognostics in Battery Health Management. IEEE Instrumentation \& Measurement Magazine, 11(4), 33-40. doi: 10.1109/MIM.2008.4579269

Goebel, K., Saha, B., Saxena, A., \& Field, M. (2008). A Comparison of Three Data-Driven Techniques For Prognostics. , 14.

Gorjian, N., Ma, L., Mittinty, M., Yarlagadda, P., \& Sun, Y. (2010). A review on degradation models in reliability analysis. In D. Kiritsis, C. Emmanouilidis, A. Koronios, \& J. Mathew (Eds.), Engineering Asset Lifecycle Management (pp. 369-384). London: Springer London. doi: 10.1007/978-0-85729-320-6 2

Guo, Y., Hou, Z., Liu, S., \& Jin, S. (2019). Data-Driven Model-Free Adaptive Predictive Control for a Class of MIMO Nonlinear Discrete-Time Systems With Stability Analysis. IEEE Access, 7, 102852-102866. doi: 10.1109/ACCESS.2019.2931198

He, W., Williard, N., Chen, C., \& Pecht, M. (2014a). State of charge estimation for li-ion batteries using neural network modeling and unscented kalman filter-based error cancellation. International Journal of Electrical Power and Energy Systems, 62, 783 - 791. doi: https://doi.org/10.1016/j.ijepes.2014.04.059

He, W., Williard, N., Chen, C., \& Pecht, M. (2014b). State of charge estimation for li-ion batteries using neural network modeling and unscented kalman filter-based error cancellation. International Journal of Electrical Power \& Energy Systems, 62, 783 - 791. doi: https://doi.org/10.1016/j.ijepes.2014.04.059

Heimes, F. O. (2008, October). Recurrent neural networks for remaining useful life estimation. In 2008 International Conference on Prognostics and Health Management (pp. 1-6). Denver, CO, USA: IEEE. doi: 10.1109/PHM.2008.4711422

Hjalmarsson, H. (2002). Iterative feedback tuningan overview. International Journal of Adaptive Control and Signal Processing, 16(5), 373-395. doi: 10.1002/acs.714

Holzer, W. (2011). A380 Advanced Cabin Line Maintenance. , 41.

Hou, Z., \& Jin, S. (2011, Nov). A novel data-driven control approach for a class of discrete-time nonlinear systems. IEEE Transactions on Control Systems Technology, 19(6), 1549-1558. doi: 10.1109/TCST.2010.2093136

Hou, Z., \& Jin, S. (2014). Model Free Adaptive Control. , 59.

Howard, L., Mesick, J., Reuter, R., \& Roemer, M. (2001). An Evolvable Tri-Reasoner IVHMl System. , 15. 
IATA. (2018, June). Fact Sheet Fuel. IATA.

Incident: France A388 over Greenland on Sep 30th 2017, uncontained engine failure, fan and engine inlet separated. (n.d.).

International Civil Aviation Organization, C. A. S. o. t. W., \& staff estimates., I. (n.d.). Air passengers carried include both domestic and international aircraft passengers of air carriers registered in the country. (Tech. Rep.).

Itier, J.-B. (2007). ARTIST2 IMA \& ADCN. , 45.

Jafari, M., Khan, K., \& Gauchia, L. (2018, December). Deterministic models of Li-ion battery aging: It is a matter of scale. Journal of Energy Storage, 20, 67-77. doi: 10.1016/j.est.2018.09.002

Jaoude, A. A. (2015). Analytic and linear prognostic model for a vehicle suspension system subject to fatigue. Systems Science \& Control Engineering, 3(1), 81-98. doi: 10.1080/21642583.2014.987359

Jardine, A. K., Lin, D., \& Banjevic, D. (2006, October). A review on machinery diagnostics and prognostics implementing condition-based maintenance. Mechanical Systems and Signal Processing, 20(7), 1483-1510. doi: 10.1016/j.ymssp.2005.09.012

Javed, G. R. Z. N., K. (2013, November). Novel failure prognostics approach with dynamic thresholds for machine degradation. In IECON 2013 - 39th Annual Conference of the IEEE Industrial Electronics Society (pp. 4404-4409). Vienna, Austria: IEEE. doi: 10.1109/IECON.2013.6699844

Jeong, J., Kim, N., Lim, W., Park, Y.-I., Cha, S. W., \& Jang, M. E. (2017, October). Optimization of power management among an engine, battery and ultra-capacitor for a series HEV: A dynamic programming application. International Journal of Automotive Technology, 18(5), 891-900. doi: 10.1007/s12239-017-0087-4

Juesas, P., Ramasso, E., Drujont, S., \& Placet, V. (2016). On partially supervised learning and inference in dynamic Bayesian networks for prognostics with uncertain factual evidence: Illustration with Markov switching models., 10 .

Jung, W., \& Ismail, A. (2015). Prognostic and Health Management Trend in Automotive Industry: An Overview. , 7 .

Kan, M. S., Tan, A. C., \& Mathew, J. (2015, October). A review on prognostic techniques for nonstationary and non-linear rotating systems. Mechanical Systems and Signal Processing, 62-63, 1-20. doi: 10.1016/j.ymssp.2015.02.016

Klabfleisch, J. D., \& Prentice, R. L. (2002). The statistical analysis of failure time data, 2nd edition. New York, USA: Wiley.

Ko, T., Karayel, D., Boru, B., Ayhan, V., Cesur, ., \& Parlak, A. (2014, January). Design and Implementation of the Control System of an Internal Combustion Engine Test
Unit. Advances in Mechanical Engineering, 6, 914876. doi: 10.1155/2014/914876

Lasheras, F., Nieto, P., de Cos Juez, F., Bayn, R., \& Surez, V. (2015, March). A Hybrid PCA-CART-MARS-Based Prognostic Approach of the Remaining Useful Life for Aircraft Engines. Sensors, 15(3), 7062-7083. doi: $10.3390 / \mathrm{s} 150307062$

Lee, J., Wu, F., Zhao, W., Ghaffari, M., Liao, L., \& Siegel, D. (2014, January). Prognostics and health management design for rotary machinery systemsReviews, methodology and applications. Mechanical Systems and Signal Processing, 42(1-2), 314-334. doi: 10.1016/j.ymssp.2013.06.004

Li, R., Verhagen, W. J. C., \& Curran, R. (2018). A Functional Architecture of Prognostics and Health Management using a Systems Engineering Approach., 10.

Li, S., Zhang, G., \& Wang, J. (2017, June). Civil aircraft health management research based on big data and deep learning technologies. In 2017 IEEE International Conference on Prognostics and Health Management (ICPHM) (pp. 154-159). Dallas, TX, USA: IEEE. doi: 10.1109/ICPHM.2017.7998321

Li, X., Ding, Q., \& Sun, J.-Q. (2018, April). Remaining useful life estimation in prognostics using deep convolution neural networks. Reliability Engineering \& System Safety, 172, 1-11. doi: 10.1016/j.ress.2017.11.021

Lin, W.-C., \& Ghoneim, Y. A. (2016, June). Model-based fault diagnosis and prognosis for Electric Power Steering systems. In 2016 IEEE International Conference on Prognostics and Health Management (ICPHM) (pp. 1-8). Ottawa, ON, Canada: IEEE. doi: 10.1109/ICPHM.2016.7542840

Lipton, Z. C. (2015). A Critical Review of Recurrent Neural Networks for Sequence Learning. , 34.

Lipu, M. H., Hannan, M., Hussain, A., Hoque, M., Ker, P. J., Saad, M., \& Ayob, A. (2018, December). A review of state of health and remaining useful life estimation methods for lithium-ion battery in electric vehicles: Challenges and recommendations. Journal of Cleaner Production, 205, 115-133. doi: 10.1016/j.jclepro.2018.09.065

Liu, J., Wang, W., Ma, F., Yang, Y., \& Yang, C. (2012). A data-model-fusion prognostic framework for dynamic system state forecasting. Engineering Applications of Artificial Intelligence, 25(4), 814 - 823. (Special Section: Dependable System Modelling and Analysis) doi: https://doi.org/10.1016/j.engappai.2012.02.015

Liu, W., Tang, B., Han, J., Lu, X., Hu, N., \& He, Z. (2015, April). The structure healthy condition monitoring and fault diagnosis methods in wind turbines: A review. Renewable and Sustainable Energy Reviews, 44, 466472. doi: 10.1016/j.rser.2014.12.005

Luo, H., Huang, M., \& Zhou, Z. (2018). Integration of multigaussian fitting and lstm neural networks for health 
monitoring of an automotive suspension component. Journal of Sound and Vibration, 428, 87 - 103. doi: https://doi.org/10.1016/j.jsv.2018.05.007

Luo, J., Namburu, M., Pattipati, K. R., Qiao, L., \& Chigusa, S. (2010, March). Integrated Model-Based and Data-Driven Diagnosis of Automotive Antilock Braking Systems. IEEE Transactions on Systems, Man, and Cybernetics - Part A: Systems and Humans, 40(2), 321-336. doi: 10.1109/TSMCA.2009.2034481

Luo, J., Pattipati, K. R., Qiao, L., \& Chigusa, S. (2008, Sept). Model-based prognostic techniques applied to a suspension system. IEEE Transactions on Systems, Man, and Cybernetics - Part A: Systems and Humans, 38(5), 1156-1168. doi: 10.1109/TSMCA.2008.2001055

Ma, M. J., Lu, C., Zerhouni, M. N., \& Cheng, Y. (2018). Aircraft Engine Health State Classification Using Stacked Denoising Autoencoder. , 6.

Matsuishi, M., \& Endo, T. (1968). Fatigue of metals subjected to varying stress. Japan Soc. Mech. Engineering.

Meinhold, R. J., \& Singpurwalla, N. D. (1983). Understanding the Kalman Filter.

Meneghetti, L., Terzi, M., Del Favero, S., Susto, G. A., \& Cobelli, C. (2018). Data-Driven Anomaly Recognition for Unsupervised Model-Free Fault Detection in Artificial Pancreas. IEEE Transactions on Control Systems Technology, 1-15. doi: 10.1109/TCST.2018.2885963

Miner, M. (1945). Cumulative damage in fatigue. Journal of Applied Mechanics, 12, A159-A164.

Ming Yu, \& Danwei Wang. (2014, July). Model-Based Health Monitoring for a Vehicle Steering System With Multiple Faults of Unknown Types. IEEE Transactions on Industrial Electronics, 61(7), 3574-3586. doi: 10.1109/TIE.2013.2281159

Naderi, E., Meskin, N., \& Khorasani, K. (2011). Nonlinear Fault Diagnosis of Jet Engines by Using a Multiple Model-Based Approach. , 13.

Naderi, E., Meskin, N., \& Khorasani, K. (2012). Nonlinear Fault Diagnosis of Jet Engines by Using a Multiple Model-Based Approach. Journal of Engineering for Gas Turbines and Power, 134(1), 011602. doi: 10.1115/1.4004152

Nguyen, D. V., Limmer, S., Yang, K., Olhofer, M., \& Bäck, T. (2019). Modelling and prediction of remaining useful lifetime for maintenance scheduling optimization of a car fleet. International Journal of Performability Engineering.

Ordez, C., Snchez Lasheras, F., Roca-Pardias, J., \& Juez, F. J. d. C. (2019, January). A hybrid ARIMASVM model for the study of the remaining useful life of aircraft engines. Journal of Computational and Applied Mathematics, 346, 184-191. doi: 10.1016/j.cam.2018.07.008

Paul, S., Kapoor, K., Jasani, D., Dudhwewala, R., Gowda,
V. B., \& Nair, T. R. G. (2008). Application of Artificial Neural Networks in Aircraft Maintenance, Repair and Overhaul Solutions. , 7.

Paul A. Gagniuc. (2017). Markov Chains: From Theory to Implementation and Experimentation. USA, NJ: John Wiley \& Sons.

Pecht, M., \& Jaai, R. (2010, March). A prognostics and health management roadmap for information and electronicsrich systems. Microelectronics Reliability, 50(3), 317323. doi: 10.1016/j.microrel.2010.01.006

Pecht, M., \& Jie Gu. (2009, June). Physics-of-failure-based prognostics for electronic products. Transactions of the Institute of Measurement and Control, 31(3-4), 309322. doi: 10.1177/0142331208092031

Poritz, A. (1982). Linear predictive hidden Markov models and the speech signal. In ICASSP '82. IEEE International Conference on Acoustics, Speech, and Signal Processing (Vol. 7, pp. 1291-1294). Paris, France: Institute of Electrical and Electronics Engineers. doi: 10.1109/ICASSP.1982.1171633

Rabiner, L. R. (1990). Readings in speech recognition. In A. Waibel \& K.-F. Lee (Eds.), (pp. 267-296).

Radac, M., Precup, R., \& Petriu, E. M. (2015, Nov). Modelfree primitive-based iterative learning control approach to trajectory tracking of mimo systems with experimental validation. IEEE Transactions on Neural Networks and Learning Systems, 26(11), 2925-2938. doi: 10.1109/TNNLS.2015.2460258

Radac, M.-B., \& Precup, R.-E. (2017, January). Data-driven model-free slip control of anti-lock braking systems using reinforcement Q-learning. Neurocomputing, 275, 317-329. doi: 10.1016/j.neucom.2017.08.036

Radac, M.-B., Precup, R.-E., \& Roman, R.-C. (2018, February). Data-driven model reference control of MIMO vertical tank systems with model-free VRFT and Q-Learning. ISA Transactions, 73, 227-238. doi: 10.1016/j.isatra.2018.01.014

Ramasso, E. (2014). Investigating computational geometry for failure prognostics. , 18.

Ramasso, E., \& Saxena, A. (2014). Performance Benchmarking and Analysis of Prognostic Methods for CMAPSS Datasets. , 15.

Razavi-Far, R., Chakrabarti, S., Saif, M., \& Zio, E. (2019, January). An integrated imputation-prediction scheme for prognostics of battery data with missing observations. Expert Systems with Applications, 115, 709-723. doi: 10.1016/j.eswa.2018.08.033

Rezvanizaniani, S. M., Liu, Z., Chen, Y., \& Lee, J. (2014, June). Review and recent advances in battery health monitoring and prognostics technologies for electric vehicle (EV) safety and mobility. Journal of Power Sources, 256, 110-124. doi: 10.1016/j.jpowsour.2014.01.085

Ross, T. J. (2010). Fuzzy logic with engineering applications 
(3rd ed ed.). Chichester, U.K: John Wiley. (OCLC: ocn430736639)

Saimurugan, M., Praveenkumar, T., Sabhrish, B., Sachin Menon, P., \& Sanjiv, S. (2016, September). On-Road Testing of A Vehicle for Gearbox Fault Detection using Vibration Signals. Indian Journal of Science and Technology, 9(34). doi: 10.17485/ijst/2016/v9i34/100957

Sankavaram, C., Kodali, A., Pattipati, K., Singh, S., Zhang, Y., \& Salman, M. (2016). An Inference-based Prognostic Framework for Health Management of Automotive Systems. , 16.

Sankavaram, C., Pattipati, B., Kodali, A., Pattipati, K., Azam, M., Kumar, S., \& Pecht, M. (2009, August). Model-based and data-driven prognosis of automotive and electronic systems. In 2009 IEEE International Conference on Automation Science and Engineering (pp. 96-101). Bangalore, India: IEEE. doi: 10.1109/COASE.2009.5234108

Saxena, A., Celaya, J., Balaban, E., Goebel, K., Saha, B., Saha, S., \& Schwabacher, M. (2008, October). Metrics for evaluating performance of prognostic techniques. In 2008 International Conference on Prognostics and Health Management (pp. 1-17). Denver, CO, USA: IEEE. doi: 10.1109/PHM.2008.4711436

Saxena, A., Celaya, J., Saha, B., Saha, S., \& Goebel, K. (2009, March). Evaluating algorithm performance metrics tailored for prognostics. In 2009 IEEE Aerospace conference (pp. 1-13). Big Sky, MT, USA: IEEE. doi: 10.1109/AERO.2009.4839666

Saxena, A., Celaya, J., Saha, B., Saha, S., \& Goebel, K. (2010, March). Evaluating prognostics performance for algorithms incorporating uncertainty estimates. In 2010 IEEE Aerospace Conference (pp. 1-11). Big Sky, MT: IEEE. doi: 10.1109/AERO.2010.5446828

Saxena, A., \& Goebel, K. (2008). C-mapss data set. NASA Ames Prognostics Data Repository.

Selvi, D., Piga, D., \& Bemporad, A. (2018, June). Towards direct data-driven model-free design of optimal controllers. In 2018 European Control Conference (ECC) (pp. 2836-2841). Limassol: IEEE. doi: 10.23919/ECC.2018.8550184

Shafi, U., Safi, A., Shahid, A. R., Ziauddin, S., \& Saleem, M. Q. (2018). Vehicle Remote Health Monitoring and Prognostic Maintenance System. Journal of Advanced Transportation, 2018, 1-10. doi: $10.1155 / 2018 / 8061514$

Shao, Y., Liang, J., Gu, F., Chen, Z., \& Ball, A. (2011, July). Fault Prognosis and Diagnosis of an Automotive Rear Axle Gear Using a RBF-BP Neural Network. Journal of Physics: Conference Series, 305, 012063. doi: 10.1088/1742-6596/305/1/012063

Shih, T. I.-P., \& Yang, V. (Eds.). (2014). Turbine aerodynamics, heat transfer, materials, and mechanics (No. 243).
Reston, Va: American Inst. of Aeronautics and Astronautics, Inc. (OCLC: 903312698)

Shufen, Q., \& Wanying, Z. (2013). Prognostic and Health Management System based on Flight Data. , 3.

Si, X.-S., Wang, W., Hu, C.-H., \& Zhou, D.-H. (2011, August). Remaining useful life estimation A review on the statistical data driven approaches. European Journal of Operational Research, 213(1), 1-14. doi: 10.1016/j.ejor.2010.11.018

Sikorska, J., Hodkiewicz, M., \& Ma, L. (2011a). Prognostic modelling options for remaining useful life estimation by industry. Mechanical Systems and Signal Processing, 25(5), 1803-1836. doi: 10.1016/j.ymssp.2010.11.018

Sikorska, J., Hodkiewicz, M., \& Ma, L. (2011b, July). Prognostic modelling options for remaining useful life estimation by industry. Mechanical Systems and Signal Processing, 25(5), 1803-1836. doi: 10.1016/j.ymssp.2010.11.018

Simon, D. (2008, June). A comparison of filtering approaches for aircraft engine health estimation. Aerospace Science and Technology, 12(4), 276-284. doi: 10.1016/j.ast.2007.06.002

Singh, S., Kodali, A., \& Pattipati, K. (2009, Aug). A factorial hidden markov model (fhmm)-based reasoner for diagnosing multiple intermittent faults. In 2009 ieee international conference on automation science and engineering (p. 146-151). doi: 10.1109/COASE.2009.5234134

Sobczyk, K., \& Spencer, B. (1993). Random fatigue: From data to theory. San Diego, USA: San Diego, CA: Academic.

Spall, J. C., \& Cristion, J. A. (1998, Sep.). Model-free control of nonlinear stochastic systems with discrete-time measurements. IEEE Transactions on Automatic Control, 43(9), 1198-1210. doi: 10.1109/9.718605

Stainier, L., Leygue, A., \& Ortiz, M. (2019, August). ModelFree Data-Driven Methods in Mechanics: Material Data Identification and Solvers. Computational Mechanics, 64(2), 381-393. (arXiv: 1903.07983) doi: 10.1007/s00466-019-01731-1

Su, S., Zhang, W., \& Zhao, S. (2014). Fault Prediction for Nonlinear System Using Sliding ARMA Combined with Online LS-SVR. Mathematical Problems in Engineering, 2014, 1-9. doi: 10.1155/2014/692848

Sutharssan, T., Stoyanov, S., Bailey, C., \& Yin, C. (2015, July). Prognostic and health management for engineering systems: a review of the data-driven approach and algorithms. The Journal of Engineering, 2015(7), 215222. doi: 10.1049/joe.2014.0303

Tahan, M., Tsoutsanis, E., Muhammad, M., \& Abdul Karim, Z. (2017, July). Performance-based health monitoring, diagnostics and prognostics for condition-based maintenance of gas turbines: A review. Applied Energy, 
198, 122-144. doi: 10.1016/j.apenergy.2017.04.048

Taie, M. A., Diab, M., \& ElHelw, M. (2012, October). Remote prognosis, diagnosis and maintenance for automotive architecture based on least squares support vector machine and multiple classifiers. In 2012 IV International Congress on Ultra Modern Telecommunications and Control Systems (pp. 128-134). St. Petersburg, Russia: IEEE. doi: 10.1109/ICUMT.2012.6459652

Tan, K. K., Lee, T. H., Huang, S. N., \& Leu, F. M. (2001, Apr 01). Adaptive-predictive control of a class of siso nonlinear systems. Dynamics and Control, 11(2), 151174. doi: 10.1023/A:1012583811904

Tang, L., Kacprzynski, G. J., Goebel, K., Saxena, A., Saha, B., \& Vachtsevanos, G. (2008, Oct). Prognosticsenhanced automated contingency management for advanced autonomous systems. In 2008 international conference on prognostics and health management (p. 1-9). doi: 10.1109/PHM.2008.4711448

Tian, J., Xiong, R., \& Yu, Q. (2019, February). FractionalOrder Model-Based Incremental Capacity Analysis for Degradation State Recognition of Lithium-Ion Batteries. IEEE Transactions on Industrial Electronics, 66(2), 1576-1584. doi: 10.1109/TIE.2018.2798606

Tinga, T. (2013). Springer Series in Reliability Engineering. London, Heidelberg, New York, Dordrecht: Springer.

Tipping, M. E. (1999). The Relevance Vector Machine. , 7.

Tsui, K. L., Chen, N., Zhou, Q., Hai, Y., \& Wang, W. (2015). Prognostics and Health Management: A Review on Data Driven Approaches. Mathematical Problems in Engineering, 2015, 1-17. doi: 10.1155/2015/793161

Vachtsevanos, G., Lewis, F., Roemer, M., Hess, A., \& Wu, B. (2006). Intelligent Fault Diagnosis and Prognosis for Engineering Systems: Vachtsevanos/Intelligent Fault Diagnosis. Hoboken, NJ, USA: John Wiley \& Sons, Inc. doi: 10.1002/9780470117842

Vapnik, V. N. (1995). The Nature of Statistical Learning Theory. New York, NY: Springer New York. (OCLC: 905485685)

Vatani, A., Khorasani, K., \& Meskin, N. (2015, June). Health Monitoring and Degradation Prognostics in Gas Turbine Engines Using Dynamic Neural Networks. In Volume 6: Ceramics; Controls, Diagnostics and Instrumentation; Education; Manufacturing Materials and Metallurgy; Honors and Awards (p. V006T05A030). Montreal, Quebec, Canada: ASME. doi: 10.1115/GT2015-44101

Vianna, W. O. L., Rodrigues, L. R., \& Yoneyama, T. (2015). Aircraft Line Maintenance Planning Based on PHM Data and Resources Availability Using Large Neighborhood Search. , 7.

Vogl, G. W., Weiss, B. A., \& Donmez, M. A. (2014). Standards for Prognostics and Health Management (PHM) Techniques within Manufacturing Operations. , 13.
Vogl, G. W., Weiss, B. A., \& Helu, M. (2016, Jun 09). A review of diagnostic and prognostic capabilities and best practices for manufacturing. Journal of Intelligent Manufacturing. doi: 10.1007/s10845-016-1228-8

Wang, D., Miao, Q., \& Pecht, M. (2013). Prognostics of lithium-ion batteries based on relevance vectors and a conditional three-parameter capacity degradation model. Journal of Power Sources, 239, 253 - 264. doi: https://doi.org/10.1016/j.jpowsour.2013.03.129

Wang, J., Mao, X., Zhu, K., Song, J., \& Zhuo, B. (2009, September). An intelligent diagnostic tool for electronically controlled diesel engine. Mechatronics, 19(6), 859-867. doi: 10.1016/j.mechatronics.2009.04.009

Wang, M.-H., Chao, K.-H., Sung, W.-T., \& Huang, G.-J. (2010, April). Using ENN-1 for fault recognition of automotive engine. Expert Systems with Applications, 37(4), 2943-2947. doi: 10.1016/j.eswa.2009.09.041

Wang, T., Jianbo Yu, Siegel, D., \& Lee, J. (2008, October). A similarity-based prognostics approach for Remaining Useful Life estimation of engineered systems. In 2008 International Conference on Prognostics and Health Management (pp. 1-6). Denver, CO, USA: IEEE. doi: 10.1109/PHM.2008.4711421

Wang, Y., Limmer, S., Olhofer, M., Emmerich, M. T. M., \& Bäck, T. (2019, June). Vehicle fleet maintenance scheduling optimization by multi-objective evolutionary algorithms. In 2019 ieee congress on evolutionary computation (cec) (p. 442-449). doi: 10.1109/CEC.2019.8790142

Wheeler, K. R., Kurtoglu, T., \& Poll, S. D. (2009). A Survey of Health Management User Objectives Related to Diagnostic and Prognostic Metrics. In Volume 2: 29th Computers and Information in Engineering Conference, Parts $A$ and $B$ (pp. 1287-1298). San Diego, California, USA: ASME. doi: 10.1115/DETC200987073

Wu, J. (2017). Introduction to Convolutional Neural Networks. , 31.

Xia, T., Dong, Y., Xiao, L., Du, S., Pan, E., \& Xi, L. (2018). Recent advances in prognostics and health management for advanced manufacturing paradigms. Reliability Engineering and System Safety, 178(C), 255-268. Retrieved from https: / / ideas.repec.org/a/eee/reensy/v178y2018 doi: 10.1016/j.ress.2018.06.02

Xing, Y., Ma, E. W., Tsui, K.-L., \& Pecht, M. (2013). An ensemble model for predicting the remaining useful performance of lithium-ion batteries. $\mathrm{Mi}$ croelectronics Reliability, 53(6), 811 - 820. doi: https://doi.org/10.1016/j.microrel.2012.12.003

Xu, J., Wang, Y., \& Xu, L. (2014, April). PHM-Oriented Integrated Fusion Prognostics for Aircraft Engines Based on Sensor Data. IEEE Sensors Journal, 14(4), 11241132. doi: 10.1109/JSEN.2013.2293517 
Xu, J., \& Xu, L. (2011, June). Health management based on fusion prognostics for avionics systems. Journal of Systems Engineering and Electronics, 22(3), 428-436. doi: 10.3969/j.issn.1004-4132.2011.03.010

Yang, C., Song, P., \& Liu, X. (2017, Apr 18). Failure prognostics of heavy vehicle hydro-pneumatic spring based on novel degradation feature and support vector regression. Neural Computing and Applications. doi: 10.1007/s00521-017-2986-8

Yang, F., Xing, Y., Wang, D., \& Tsui, K.-L. (2016, February). A comparative study of three model-based algorithms for estimating state-of-charge of lithiumion batteries under a new combined dynamic loading profile. Applied Energy, 164, 387-399. doi: 10.1016/j.apenergy.2015.11.072

Yang, L., Wang, J., \& Zhang, G. (2016, June). Aviation PHM system research framework based on PHM big data center. In 2016 IEEE International Conference on Prognostics and Health Management (ICPHM) (pp. 1-5). Ottawa, ON, Canada: IEEE. doi: 10.1109/ICPHM.2016.7542824

Ye, M., Guo, H., \& Cao, B. (2017, March). A model-based adaptive state of charge estimator for a lithium-ion battery using an improved adaptive particle filter. Applied Energy, 190, 740-748. doi: 10.1016/j.apenergy.2016.12.133

You, G.-w., Park, S., \& Oh, D. (2016, August). Real-time state-of-health estimation for electric vehicle batteries: A data-driven approach. Applied Energy, 176, 92-103. doi: 10.1016/j.apenergy.2016.05.051

Zhang, J., \& Lee, J. (2011, August). A review on prognostics and health monitoring of Li-ion battery. Jour- nal of Power Sources, 196(15), 6007-6014. doi: 10.1016/j.jpowsour.2011.03.101

Zhang, M. Y., Liu, J., Hanachi, D. H., Yu, M. X., \& Yang, M. Y.-B. (2018). Physics-based Model and Neural Network Model for Monitoring Starter Degradation of APU. , 7.

Zhang, X., Kang, J., Zhao, J. S., \& Cao, D. C. (2013, July). Features for fault diagnosis and prognosis of gearbox. Chemical Engineering Transactions, 1027-1032. doi: 10.3303/CET1333172

Zhao, Z., Liang, B., Wang, X., \& Lu, W. (2017). Remaining useful life prediction of aircraft engine based on degradation pattern learning. Reliability Engineering and System Safety, 164, 74 - 83. doi: https://doi.org/10.1016/j.ress.2017.02.007

Zheng, X., \& Fang, H. (2015). An integrated unscented kalman filter and relevance vector regression approach for lithium-ion battery remaining useful life and short-term capacity prediction. Reliability Engineering \& System Safety, 144, 74 - 82. doi: https://doi.org/10.1016/j.ress.2015.07.013

Zhong, S., Li, M. Z., Lin, M. L., \& Zhang, D. Y. (2018). Aero-Engine Exhaust Gas Temperature Prognostic Model Based on Gated Recurrent Unit Network. , 5 .

Zou, K.-X., Ma, H.-D., Fang, H.-Z., \& Yi, D.-W. (2011, May). Study of prognostics for spacecraft based-on particle swarm optimized neural network. In 2011 Prognostics and System Health Managment Confernece (pp. 1-5). Shenzhen, China: IEEE. doi: 10.1109/PHM.2011.5939479 